\title{
An Iterative Method for Solving Split Monotone Variational Inclusion Problems and Finite Family of Variational Inequality Problems in Hilbert Spaces
}

\author{
Wanna Sriprad (iD) and Somnuk Srisawat \\ Department of Mathematics and Computer Science, Faculty of Science and Technology, Rajamangala University of Technology, \\ Thanyaburi, Pathum Thani 12110, Thailand
}

Correspondence should be addressed to Wanna Sriprad; wanna_sriprad@rmutt.ac.th

Received 18 August 2021; Accepted 10 December 2021; Published 31 December 2021

Academic Editor: Narendra K. Govil

Copyright (c) 2021 Wanna Sriprad and Somnuk Srisawat. This is an open access article distributed under the Creative Commons Attribution License, which permits unrestricted use, distribution, and reproduction in any medium, provided the original work is properly cited.

The purpose of this paper is to study the convergence analysis of an intermixed algorithm for finding the common element of the set of solutions of split monotone variational inclusion problem (SMIV) and the set of a finite family of variational inequality problems. Under the suitable assumption, a strong convergence theorem has been proved in the framework of a real Hilbert space. In addition, by using our result, we obtain some additional results involving split convex minimization problems (SCMPs) and split feasibility problems (SFPs). Also, we give some numerical examples for supporting our main theorem.

\section{Introduction}

Let $H_{1}$ and $H_{2}$ be real Hilbert spaces whose inner product and norm are denoted by $\langle\cdot, \cdot\rangle$ and $\|\cdot\|$, respectively, and let C, Q be nonempty closed convex subsets of $\mathrm{H}_{1}$ and $\mathrm{H}_{2}$, respectively. For a mapping $S: C \longrightarrow C$, we denoted by $F(S)$ the set of fixed points of $S$ (i.e., $F(S)=\{x \in C: S x=x\}$ ). Let $A: C \longrightarrow H$ be a nonlinear mapping. The variational inequality problem (VIP) is to find $x^{*} \in C$ such that

$$
\left\langle A x^{*}, y-x^{*}\right\rangle \geq 0, \quad \forall y \in C,
$$

and the solution set of problem (1) is denoted by $V I(C, A)$. It is known that the variational inequality, as a strong and great tool, has already been investigated for an extensive class of optimization problems in economics and equilibrium problems arising in physics and many other branches of pure and applied sciences. Recall that a mapping $A: C \longrightarrow C$ is said to be $\alpha$-inverse strongly monotone if there exists $\alpha>0$ such that

$$
\langle A x-A y, x-y\rangle \geq \alpha\|A x-A y\|^{2}, \quad \forall x, y \in C .
$$

A multivalued mapping $M: H_{1} \longrightarrow 2^{H_{1}}$ is called monotone if for all $x, y \in H_{1},\langle x-y, u-v\rangle \geq 0$, for any $u \in M x$ and $v \in M y$. A monotone mapping $M: H_{1} \longrightarrow 2^{H_{1}}$ is maximal if the graph $G(M)$ for $M$ is not properly contained in the graph of any other monotone mapping. It is generally known that $M$ is maximal if and only if for $(x, u) \in H_{1} \times H_{1},\langle x-y, u-$ $v\rangle \geq 0$ for all $(y, v) \in G(M)$ implies $u \in M x$. Let $M: H_{1} \longrightarrow 2^{H_{1}}$ be a multivalued maximal monotone mapping. The resolvent mapping $J_{\lambda}^{M}: H_{1} \longrightarrow H_{1}$ associated with $M$ is defined by

$$
J_{\lambda}^{M}(x):=(I+\lambda M)^{-1}(x), \quad \forall x \in H_{1}, \lambda>0,
$$

where $I$ stands for the identity operator on $H_{1}$. We note that for all $\lambda>0$, the resolvent $J_{\lambda}^{M}$ is single-valued, nonexpansive, and firmly nonexpansive. 
In 2011, Moudafi [1] introduced the following split monotone variational inclusion problem (SMVI):

$$
\text { find } x^{*} \in H_{1} \text { such that } \theta \in A_{1}\left(x^{*}\right)+M_{1}\left(x^{*}\right)
$$

and such that $y^{*}=T x^{*} \in H_{2}$ solves $\theta \in A_{2}\left(y^{*}\right)+M_{2}\left(y^{*}\right)$,

where $\theta$ is the zero vector in $H_{1}$ and $H_{2}, M_{1}: H_{1} \longrightarrow 2^{H_{1}}$ and $M_{2}: H_{2} \longrightarrow 2^{H_{2}}$ are multivalued mappings on $H_{1}$ and $H_{2}$, $A_{1}: H_{1} \longrightarrow H_{1}$ and $A_{2}: H_{2} \longrightarrow H_{2}$ are two given singlevalued mappings, and $T: H_{1} \longrightarrow H_{2}$ is a bounded linear operator with adjoint $T^{*}$ of $T$. We note that if (4) and (5) are considered separately, we have that (4) is a variational inclusion problem with its solution set $V I\left(H_{1}, A_{1}, M_{1}\right)$ and (5) is a variational inclusion problem with its solution set $V I\left(H_{1}, A_{2}, M_{2}\right)$. We denoted the set of all solutions of (SMVI) by $\Omega=\left\{x^{*} \in V I\left(H_{1}, A_{1}, M_{1}\right): T x^{*} \in V I\left(H_{2}, A_{2}, M_{2}\right)\right\}$.

It is worth noticing that by taking $M_{1}=N_{C}$ and $M_{2}=$ $N_{Q}$ normal cones to closed convex sets $C$ and $Q$, then (SMVI) (4) and (5) reduce to the split variational inequality problem (SVIP) that was introduced by Censor et at. [2]. In [1], they mentioned that (SMVI) (4) and (5) contain many special cases, such as split minimization problem (SMP), split minimax problem (SMMP), and split equilibrium problem (SEP). Some related works can be found in [1, 3-10].

For solving (SMVI) (4) and (5), Modafi [1] proposed the following algorithm.

Algorithm 1. Let $\lambda>0, x_{0} \in H_{1}$, and the sequence $\left\{x_{n}\right\}$ be generated by

$x_{n+1}=J_{\lambda}^{M_{1}}(1-\lambda f)\left(x_{n}+\gamma T^{*}\left(J_{\lambda}^{M_{2}}(I-\lambda g)-I\right) T x_{n}\right), \quad n \in \mathbb{N}$, where $\gamma \in(1,1 / L)$ with $L$ being the spectral radius of the operator $T^{*} T$.

He obtained the following weak convergence theorem for algorithm (6).

Theorem 1 (see [1]). Let $H_{1}, H_{2}$ be real Hilbert spaces. Let $T: H_{1} \longrightarrow H_{2}$ be a bounded linear operator with adjoint $T^{*}$. For $i=1,2$, let $A_{i}: H_{i} \longrightarrow H_{i}$ be $\alpha_{i}$-inverse strongly monotone with $\alpha=\min \left\{\alpha_{1}, \alpha_{2}\right\}$ and let $M_{i}: H_{i} \longrightarrow 2^{M_{i}}$ be two maximal monotone operators. Then, the sequence generated by (6) converges weakly to an element $x^{*} \in \Omega$ provided that $\Omega \neq \varnothing, \lambda \in(0,2 \alpha)$, and $\gamma \in(1,1 / L)$ with $L$ being the spectral radius of the operator $T^{*} T$.

Since then, because of a lot of applications of (SMVI), it receives much attention from many authors. They presented many approximation methods for solving (SMVI) (4) and (5). Also the iterative methods for solving (SMVIP) (4) and (5) and fixed-point problems of some nonlinear mappings have been investigated (see [11-19]).

On the other hand, Yao et al. [20] presented an intermixed Algorithm 1.3 for two strict pseudo-contractions in real Hilbert spaces. They also showed that the suggested algorithms converge strongly to the fixed points of two strict pseudo-contractions, independently. As a special case, they can find the common fixed points of two strict pseudocontractions in Hilbert spaces (i.e., a mapping $S: C \longrightarrow C$ is said to be $\kappa$-strictly pseudo-contractive if there exists a constant $\kappa \in[0,1)$ such that $\|S x-S y\|^{2} \leq\|x-y\|^{2}+k$ $\|(I-S) x-(I-S)\|, 0.3 \mathrm{~cm} \forall x, y \in C)$.

Algorithm 2. For arbitrarily given $x_{0}, y_{0} \in C$, let the sequences $\left\{x_{n}\right\}$ and $\left\{y_{n}\right\}$ be generated iteratively by

$$
\begin{cases}x_{n+1}=\left(1-\beta_{n}\right) x_{n}+\beta_{n} P_{C}\left[\alpha_{n} f\left(y_{n}\right)+\left(1-k-\alpha_{n}\right) x_{n}+k T x_{n}\right], & n \geq 0 \\ y_{n+1}=\left(1-\beta_{n}\right) y_{n}+\beta_{n} P_{C}\left[\alpha_{n} f\left(x_{n}\right)+\left(1-k-\alpha_{n}\right) y_{n}+k S y_{n}\right], & n \geq 0\end{cases}
$$

where $\left\{\alpha_{n}\right\}$ and $\left\{\beta_{n}\right\}$ are two sequences of the real number in $(0,1), T, S: C \longrightarrow C$ are $\lambda$-strictly pseudo-contractions, $f: C \longrightarrow H$ is a $\rho_{1}$-contraction, $g: C \longrightarrow H$ is a $\rho_{2}$-contraction, and $k \in(0,1-\lambda)$ is a constant.

Under some control conditions, they proved that the sequence $\left\{x_{n}\right\}$ converges strongly to $P_{F(T)} f\left(y^{*}\right)$ and $\left\{y_{n}\right\}$ converges strongly to $P_{F(S)} f\left(x^{*}\right)$, respectively, where $x^{*} \in F(T), y^{*} \in F(S)$, and $P_{F(T)}$ and $P_{F(S)}$ are the metric projection of $H$ onto $F(T)$ and $F(S)$, respectively. After that, many authors have developed and used this algorithm to solve the fixed-point problems of many nonlinear operators in real Hilbert spaces (see for example [21-27]). Question: can we prove the strong convergence theorem of two sequences of split monotone variational inclusion problems and fixed-point problems of nonlinear mappings in real Hilbert spaces?
The purpose of this paper is to modify an intermixed algorithm to answer the question above and prove a strong convergence theorem of two sequences for finding a common element of the set of solutions of (SMVI) (4) and (5) and the set of solutions of a finite family of variational inequality problems in real Hilbert spaces. Furthermore, by applying our main result, we obtain some additional results involving split convex minimization problems (SCMPs) and split feasibility problems (SFPs). Finally, we give some numerical examples for supporting our main theorem.

\section{Preliminaries}

Let $H$ be a real Hilbert space and $C$ be a nonempty closed convex subset of $H$. We denote the strong convergence of $\left\{x_{n}\right\}$ to $x$ and the weak convergence of $\left\{x_{n}\right\}$ to $x$ by notations " $x_{n} \longrightarrow x$ as $n \longrightarrow \infty$ " and " $x_{n}-x$ as $n \longrightarrow \infty$," 
respectively. For each $x, y, z \in H$ and $\alpha, \beta, \gamma \in[0,1]$ with $\alpha+\beta+\gamma=1$, we have

$$
\begin{gathered}
\|x+y\|^{2} \leq\|x\|^{2}+2\langle y, x+y\rangle, \\
\|\alpha x+\beta y+\gamma z\|^{2}=\alpha\|x\|^{2}+\beta\|y\|^{2}+\gamma\|x\|^{2}-\alpha \beta\|x-y\|^{2}-\alpha \gamma\|x-z\|^{2}-\beta \gamma\|y-z\|^{2} . \\
u \in V I(C, A) \Leftrightarrow u=P_{C}(I-\lambda A) u,
\end{gathered}
$$

Definition 1. Let $H$ be a real Hilbert space and $C$ be a closed convex subset of $H$. Let $S: C \longrightarrow C$ be a mapping. Then, $S$ is said to be

(1) Monotone, if $\langle S x-S y, x-y\rangle \geq 0, \forall x, y \in H$

(2) Firmly nonexpansive, if $\langle S x-S y, x-y\rangle \geq \| S x-$ $S y \|^{2}, \forall x, y \in H$

(3) Lipschitz continuous, if there exists a constant $L>0$ such that $\|S x-S y\| \leq L\|x-y\|, \forall x, y \in H$

(4) Nonexpansive, if $=\|S x-S y\| \leq\|x-y\|, \forall x, y \in H$

It is well known that if $S$ is $\alpha$-inverse strongly monotone, then it is $1 / \alpha$-Lipschitz continuous and every nonexpansive mapping $S$ is 1-Lipschitz continuous. We note that if $S: H \longrightarrow H$ is a nonexpansive mapping, then it satisfies the following inequality (see Theorem 3 in [28] and Theorem 1 in [29]):

$\langle S y-S x,(I-S) x-(I-S) y\rangle \leq \frac{1}{2}\|(I-S) x-(I-S) y\|^{2}$,

$\forall x, y \in H$

Particularly, for every $x \in H$ and $y \in F(S)$, we have

$$
\langle y-S x,(I-S) x\rangle \leq \frac{1}{2}\|(I-S) x\|^{2} .
$$

For every $x \in H$, there is a unique nearest point $P_{C} x$ in $C$ such that

$$
\left\|x-P_{C} x\right\| \leq\|x-y\|, \quad \forall y \in C .
$$

Such an operator $P_{C}$ is called the metric projection of $H$ onto $C$.

Lemma 1 (see [30]). For a given $z \in H$ and $u \in C$,

$$
u=P_{C} z \Leftrightarrow\langle u-z, v-u\rangle \geq 0, \quad \forall v \in C .
$$

Furthermore, $P_{C}$ is a firmly nonexpansive mapping of $H$ onto $C$ and satisfies

$$
\left\|P_{C} x-P_{C} y\right\|^{2} \leq\left\langle P_{C} x-P_{C} y, x-y\right\rangle, \quad \forall x, y \in H .
$$

Moreover, we also have the following lemma.

Lemma 2 (see [31]). Let $H$ be a real Hilbert space, let $C$ be a nonempty closed convex subset of $H$, and let $A$ be a mapping of $C$ into $H$. Let $u \in C$. Then, for $\lambda>0$, where $P_{C}$ is the metric projection of $H$ onto $C$.

Lemma 3. Let $C$ be a nonempty closed and convex subset of a real Hilbert space $H$. For every $i=1,2, \ldots, N$, let $A_{i}: C \longrightarrow H$ be the $\alpha_{i}$-inverse strongly monotone with $\bar{\alpha}=\min _{i=1,2, \ldots, N}\left\{\alpha_{i}\right\}$. If $\cap_{i=1}^{N} V I\left(C, A_{i}\right) \neq \varnothing$, then

$$
V I\left(C, \sum_{i=1}^{N} a_{i} A_{i}\right)=\bigcap_{i=1}^{N} V I\left(C, A_{i}\right),
$$

where $0<a_{i}<1$ for all $i=1,2, \ldots, N$ and $\sum_{i=1}^{N} a_{i}=1$. Moreover, $I-\lambda \sum_{i=1}^{N} a_{i} A_{i}$ is a nonexpansive mapping for all $\lambda \in(0,2 \bar{\alpha})$.

Proof. By Lemma 4.3 of [32], we have that $V I\left(C, \sum_{i=1}^{N} a_{i} A_{i}\right)=\cap_{i=1}^{N} V I\left(C, A_{i}\right)$. Let $\lambda \in(0,2 \bar{\alpha})$ and let $x, y \in C$. As the same argument as in the proof of Lemma 8 in [16], we have $I-\lambda \sum_{i=1}^{N} a_{i} A_{i}$ as nonexpansive.

Lemma 4 (see [33]). Let $H$ be a real Hilbert space, $A: H \longrightarrow H$ be a single-valued nonlinear mapping, and $M: H \longrightarrow 2^{H}$ be a set-valued mapping. Then, a point $u \in H$ is a solution of variational inclusion problem if and only if $u=J_{\lambda}^{M}(I-\lambda A) u, \forall \lambda>0$, i.e.,

$$
V I(H, A, M)=F\left(J_{\lambda}^{M}(I-\lambda A)\right), \quad \forall \lambda>0 .
$$

Furthermore, if $A$ is $\alpha$-inverse strongly monotone and $\lambda \in(0,2 \alpha]$, then $V I(H, A, M)$ is a closed convex subset of $H$.

Lemma 5 (see [33]). ie resolvent operator $J_{\lambda}^{M}$ associated with $M$ is single-valued, nonexpansive, and 1-inverse strongly monotone for all $\lambda>0$.

The following two lemmas are the particular case of Lemmas 7 and 8 in [16].

Lemma 6 (see [16]). For every $i=1,2$, let $H_{i}$ be real Hilbert spaces, let $M_{i}: H_{i} \longrightarrow 2^{H_{i}}$ be a multivalued maximal monotone mapping, and let $A_{i}: H_{i} \longrightarrow H_{i}$ be an $\alpha_{i}$-inverse strongly monotone mapping. Let $\mathrm{T}: \mathrm{H}_{1} \longrightarrow \mathrm{H}_{2}$ be a bounded linear operator with adjoint $T^{*}$ of $T$, and let $\widetilde{G}: H_{1} \longrightarrow H_{1}$ be a mapping defined by $\widetilde{G}(x)=J_{\lambda_{1}}^{M_{1}}\left(I-\lambda_{1} A_{1}\right)\left(x-\gamma T^{*}\right.$ $\left.\left(I-J_{\lambda_{2}}^{M_{2}}\left(I-\lambda_{2} A_{2}\right)\right) T x\right)$, for all $x \in H_{1}$. Then, $\| \widetilde{G} x-$ $\widetilde{G} y\left\|^{2} \leq\right\| x-y\left\|^{2}-\gamma(1-\gamma L)\right\|\left(I-J_{\lambda_{2}}^{M_{2}}\left(I-\lambda_{2} A_{2}\right)\right) T x-(I-$ $\left.J_{\lambda_{2}}^{M_{2}}\left(I-\lambda_{2} A_{2}\right)\right) T y \|^{2}$, for all $x, y \in H_{1}$, where $L$ is the spectral radius of the operator $T^{*} T, \lambda_{1} \in\left(0,2 \alpha_{1}\right), \lambda_{2} \in\left(0,2 \alpha_{2}\right)$, and $\gamma>0$. Furthermore, if $0<\gamma<1 / L$, then $\widetilde{G}$ is a nonexpansive mapping. 
Lemma 7 (see [16]). Let $H_{1}$ and $H_{2}$ be Hilbert spaces. For $i=1,2$, let $M_{i}: H_{i} \longrightarrow 2^{H_{i}}$ be a multivalued maximal monotone mapping and let $A_{i}: H_{i} \longrightarrow H_{i}$ be an $\alpha_{i}$-inverse strongly monotone mapping. Let $T: H_{1} \longrightarrow H_{2}$ be a bounded linear operator with adjoint $T^{*}$. Assume that $\Omega \neq \phi$. Then, $x^{*} \in \Omega$ if and only if $x^{*}=\widetilde{G}\left(x^{*}\right)$, where $\widetilde{G}: H_{1} \longrightarrow H_{1}$ is a mapping defined by

$\widetilde{G}(x)=J_{\lambda_{1}}^{M_{1}}\left(I-\lambda_{1} A_{1}\right)\left(x-\gamma T^{*}\left(I-J_{\lambda_{2}}^{M_{2}}\left(I-\lambda_{2} A_{2}\right)\right) T x\right)$,

for all $\quad x \in H_{1}, \quad \lambda_{1} \in\left(0,2 \alpha_{1}\right), \lambda_{2} \in\left(0,2 \alpha_{2}\right), \quad$ and $0<\gamma<1 / L$, where $L$ is the spectral radius of the operator $T^{*} T$.

Next, we give an example to support Lemma 7.

Example 1. Let $\mathbb{R}$ be a set of real number and $H_{1}=H_{2}=\mathbb{R}^{2}$, and let $\langle\cdot, \cdot\rangle: \mathbb{R}^{2} \times \mathbb{R}^{2} \longrightarrow \mathbb{R}$ be inner product defined by $\langle x, y\rangle=x \cdot y=x_{1} y_{1}+x_{2} y_{2}$, for all $x=\left(x_{1}, x_{2}\right) \in \mathbb{R}^{2}$ and $y=\left(y_{1}, y_{2}\right) \in \mathbb{R}^{2}$ and the usual norm $\|\cdot\|: \mathbb{R}^{2} \longrightarrow \mathbb{R}$ given by $\|x\|=\sqrt{x_{1}^{2}+x_{2}^{2}}$, for all $x=\left(x_{1}, x_{2}\right) \in \mathbb{R}^{2}$. Let T: $H_{1} \longrightarrow H_{2}$ be defined by $T x=\left(2 x_{1}, 2 x_{2}\right)$ for all $x=$ $\left(x_{1}, x_{2}\right) \in \mathbb{R}^{2}$ and $T^{*}: \mathbb{R}^{2} \longrightarrow \mathbb{R}^{2}$ be defined by $T^{*} z=\left(2 z_{1}, 2 z_{2}\right)$ for all $z=\left(z_{1}, z_{2}\right) \in \mathbb{R}^{2}$. Let $M_{1}, M_{2}$ : $\mathbb{R}^{2} \longrightarrow 2^{\mathbb{R}^{2}}$ be defined by $M_{1} x=\left\{\left(3 x_{1}-5,3 x_{2}-\right.\right.$ 5) $\}$ and $M_{2} x=\left\{\left(x_{1} / 3-2, x_{2} / 3-2\right)\right\}$, respectively, for all $x=\left(x_{1}, x_{2}\right) \in \mathbb{R}^{2}$. Let the mapping $A_{1}, A_{2}: \mathbb{R}^{2} \longrightarrow \mathbb{R}^{2}$ be defined by $A_{1} x=\left(\left(x_{1}-4\right) / 2,\left(x_{2}-4\right) / 2\right)$ and $A_{2} x=\left(\left(x_{1}-\right.\right.$ $\left.2) / 3,\left(x_{2}-2\right) / 3\right)$, respectively, for all $x=\left(x_{1}, x_{2}\right) \in \mathbb{R}^{2}$. Then, $(2,2)$ is a fixed point of $\widetilde{G}$. That is, $(2,2) \in F(\widetilde{G})$.

Proof. It is obvious to see that $\Omega=\{(2,2)\}, A_{1}$ is 2-inverse strongly monotone, and $A_{2}$ is 3 -inverse strongly monotone. Choose $\lambda_{1}=1 / 3$. Since $M_{1} x=\left\{\left(3 x_{1}-5,3 x_{2}-5\right)\right\}$ and the resolvent of $M_{1}, J_{\lambda_{1}}^{M_{1}^{1}} x=\left(I+\lambda_{1} M_{1}\right)^{-1} x$ for all $x=\left(x_{1}, x_{2}\right) \in \mathbb{R}^{2}$, we obtain that

$$
J_{\lambda_{1}}^{M_{1}} x=\frac{x}{2}+\frac{5}{6}, \quad \text { for all } x=\left(x_{1}, x_{2}\right) \in \mathbb{R}^{2} .
$$

Choose $\lambda_{2}=1$. Since $M_{2} x=\left\{\left(x_{1} / 3-2, x_{2} / 3-2\right)\right\}$ and the resolvent of $M_{2}, J_{\lambda_{2}}^{M_{2}} x=\left(I+\lambda_{2} M_{2}\right)^{-1} x$ for all $x=\left(x_{1}, x_{2}\right) \in \mathbb{R}^{2}$, we obtain that

$$
J_{\lambda_{2}}^{M_{2}} x=\frac{3 x}{4}+\frac{3}{2}, \quad \text { for all } x=\left(x_{1}, x_{2}\right) \in \mathbb{R}^{2} .
$$

Since the spectral radius of the operator $T^{*} T$ is 4 , we choose $\gamma=0.1$. Then, from (18) and (19), we get that

$$
\begin{aligned}
\widetilde{G}(x) & =J_{1 / 3}^{M_{1}}\left(I-\frac{1}{3} A_{1}\right)\left(x-0.1 T^{*}\left(I-J_{1}^{M_{2}}\left(I-A_{2}\right)\right) T x\right), \\
& =\frac{x}{3}+\frac{4}{3},
\end{aligned}
$$

for all $x=\left(x_{1}, x_{2}\right) \in \mathbb{R}^{2}$. Then, by Lemma 7 , we have that $(2,2) \in F(\widetilde{G})$.

Lemma 8 (see [34]). Let $\left\{s_{n}\right\}$ be a sequence of nonnegative real numbers satisfying $s_{n+1} \leq\left(1-\alpha_{n}\right) s_{n}+\delta_{n}, \forall n \geq 0$ where $\left\{\alpha_{n}\right\}$ is a sequence in $(0,1)$ and $\left\{\delta_{n}\right\}$ is a sequence in $\mathbb{R}$ such that

(1) $\sum_{n=0}^{\infty} \alpha_{n}=\infty$.

(2) $\limsup \operatorname{si\infty }_{n} \alpha_{n} / \delta_{n} \leq 0 \quad$ or $\quad \sum_{n=0}^{\infty}\left|\delta_{n}\right|<\infty$. Then $\lim _{n \longrightarrow \infty} s_{n}=0$.

\section{Main Results}

In this section, we introduce an iterative algorithm of two sequences which depend on each other by using the intermixed method. Then, we prove a strong convergence theorem for solving two split monotone variational inclusion problems and a finite family of variational inequality problems.

Theorem 2. Let $H_{1}$ and $H_{2}$ be Hilbert spaces, and let $C$ be a nonempty closed convex subset of $H_{1}$. Let T: $H_{1} \longrightarrow H_{2}$ be a bounded linear operator, and let $f, g: H_{1} \longrightarrow H_{1}$ be $\rho_{f}, \rho_{g}$-contraction mappings with $\rho=\max \left\{\rho_{f}, \rho_{g}\right\}$. For $i=1,2$, let $M_{i}^{x}, M_{i}^{y}: H_{i} \longrightarrow 2^{H_{i}}$ be multivalued maximal monotone mappings and let $A_{i}^{x}, A_{i}^{y}: H_{i} \longrightarrow H_{i}$ be $\alpha_{i}^{x}, \alpha_{i}^{y}$-inverse strongly monotone mappings, respectively. For $i=1,2, \ldots, N$, let $B_{i}^{x}, B_{i}^{y}: H_{1} \longrightarrow H_{1}$ be $\beta_{i}^{x}, \beta_{i}^{y}$-inverse strongly monotone mappings, respectively, $\bar{\beta}_{x}=$ $\min _{i=1,2, \ldots, N}\left\{\beta_{i}^{x}\right\}$, and $\bar{\beta}_{y}=\min _{i=1,2, \ldots, N}\left\{\beta_{i}^{y}\right\}$. Let $\widetilde{G}^{x}, \widetilde{G}^{y}$ : $H_{1} \longrightarrow H_{1}$ be defined by $\widetilde{G}^{x} x=J_{\lambda_{1}^{x}}^{M_{1}^{x}}\left(I-\lambda_{1}^{x} A_{1}^{x}\right)(x-$ $\left.\gamma^{x} T^{*}\left(I-J_{\lambda_{2}^{x}}^{M_{2}^{x}}\left(I-\lambda_{2}^{x} A_{2}^{x}\right)\right) T x\right), \forall x \in H_{1}$, and $\widetilde{G}^{y} y=J_{\lambda_{1}^{y}}^{M_{1}^{y}}(I-$ $\left.\lambda_{1}^{y} A_{1}^{y}\right)\left(y-\gamma^{y} T^{*}\left(I-J_{\lambda_{2}^{y}}^{M_{2}^{y}}\left(I-\lambda_{2}^{y} A_{2}^{y}\right)\right) T y\right), \forall y \in H_{1}$, respectively, where $\lambda_{i}^{x} \in\left(0,2 \alpha_{i}^{x}\right), \lambda_{i}^{y} \in\left(0,2 \alpha_{i}^{y}\right)$, and $0<\gamma^{x}, \gamma^{y}<1 / L$ with $L$ being a spectral radius of $T^{*} T$. Assume that $\mathscr{F}^{x}=$ $\Omega^{x} \cap\left(\cap_{i=1}^{N} V I\left(C, B_{i}^{x}\right)\right) \neq \varnothing \quad$ and $\mathscr{F}^{y}=\Omega^{y} \cap\left(\cap_{i=1}^{N} V I(C\right.$, $\left.\left.B_{i}^{y}\right)\right) \neq \varnothing$. Let $\left\{x_{n}\right\}$ and $\left\{y_{n}\right\}$ be sequences generated by $x_{1}, y_{1} \in H_{1}$ and

$$
\left\{\begin{array}{l}
x_{n+1}=\delta_{n} x_{n}+\sigma_{n} P_{C}\left(I-\mu_{n}^{x} \sum_{i=1}^{N} a_{i}^{x} B_{i}^{x}\right) x_{n}+\eta_{n}\left(\alpha_{n} f\left(y_{n}\right)+\left(1-\alpha_{n}\right) \widetilde{G}^{x} x_{n}\right), \\
y_{n+1}=\delta_{n} y_{n}+\sigma_{n} P_{C}\left(I-\mu_{n}^{y} \sum_{i=1}^{N} a_{i}^{y} B_{i}^{y}\right) y_{n}+\eta_{n}\left(\alpha_{n} g\left(x_{n}\right)+\left(1-\alpha_{n}\right) \widetilde{G}^{y} y_{n}\right),
\end{array}\right.
$$


for all $n \geq 1$ where $\left\{\delta_{n}\right\},\left\{\sigma_{n}\right\},\left\{\eta_{n}\right\},\left\{\alpha_{n}\right\} \subseteq[0,1]$ with $\delta_{n}+\sigma_{n}+\eta_{n}=1, \quad\left\{a_{1}^{x}, a_{2}^{x}, \ldots, a_{N}^{x}\right\},\left\{a_{1}^{y}, a_{2}^{y}, \ldots, a_{N}^{y}\right\} \subset(0,1)$, and $\left\{\mu_{n}^{x}\right\},\left\{\mu_{n}^{y}\right\} \subset(0, \infty)$. Assume the following condition holds:

(1) $\sum_{n=1}^{\infty} \mu_{n}^{x}<\infty, \sum_{n=1}^{\infty} \mu_{n}^{y}<\infty$, $\quad$ and $0<a<\mu_{n}^{x} \leq 2 \bar{\beta}_{x}, 0<b<\mu_{n}^{y} \leq 2 \overline{\beta_{y}}$, for some $a, b \in \mathbb{R}$.

(2) $\sum_{n=1}^{\infty} \alpha_{n}=\infty, \lim _{n \longrightarrow \infty} \alpha_{n}=0$.

(3) $\sum_{n=1}^{N} a_{i}^{x}=\sum_{n=1}^{N} a_{i}^{y}=1$.

(4) $0<\bar{a} \leq \delta_{n}, \sigma_{n}, \eta_{n} \leq \bar{b}<1$, for all $n \in \mathbb{N}$, for some $\bar{a}, \bar{b}>0$.
(5) $\sum_{n=1}^{\infty}\left|\delta_{n+1}-\delta_{n}\right|, \sum_{n=1}^{\infty}\left|\sigma_{n+1}-\sigma_{n}\right|$,

and $\sum_{n=1}^{\infty}\left|\alpha_{n+1}-\alpha_{n}\right|<\infty$. Then, $\left\{x_{n}\right\}$ converges strongly to $\tilde{x}=P_{\mathscr{F}^{x}} f(\tilde{y})$ and $\left\{y_{n}\right\}$ converges strongly to $\tilde{y}=P_{\mathscr{F}^{y}} g(\tilde{x})$.

Proof. We divided the proof into five steps.

Step 1. We will show that $\left\{x_{n}\right\}$ and $\left\{y_{n}\right\}$ are bounded. Let $x^{*} \in \mathscr{F}^{x}$ and $y^{*} \in \mathscr{F}^{y}$. Then, from Lemma 7 and Lemma 6 , we get

$$
\begin{aligned}
\left\|\widetilde{G}^{x} x_{n}-x^{*}\right\| & =\left\|J_{\lambda_{1}^{x}}^{M_{1}^{x}}\left(I-\lambda_{1}^{x} A_{1}^{x}\right)\left(x_{n}-\gamma^{x} T^{*}\left(I-J_{\lambda_{2}^{x}}^{M_{2}^{x}}\left(I-\lambda_{2}^{x} A_{2}^{x}\right)\right) T x_{n}\right)-x^{*}\right\|, \\
& \leq\left\|x_{n}-x^{*}\right\| .
\end{aligned}
$$

From (21), Lemma 3, and (22), we have

$$
\begin{aligned}
\left\|x_{n+1}-x^{*}\right\| & =\left\|\delta_{n} x_{n}+\sigma_{n} P_{C}\left(I-\mu_{n}^{x} \sum_{i=1}^{N} a_{i}^{x} B_{i}^{x}\right) x_{n}+\eta_{n}\left(\alpha_{n} f\left(y_{n}\right)+\left(1-\alpha_{n}\right) \widetilde{G}^{x} x_{n}\right)-x^{*}\right\|, \\
& \leq \delta_{n}\left\|x_{n}-x^{*}\right\|+\sigma_{n}\left\|P_{C}\left(I-\mu_{n}^{x} \sum_{i=1}^{N} a_{i}^{x} B_{i}^{x}\right) x_{n}-x^{*}\right\|+\eta_{n}\left\|\left(\alpha_{n} f\left(y_{n}\right)+\left(1-\alpha_{n}\right) \widetilde{G}^{x} x_{n}\right)-x^{*}\right\| \\
& \leq \delta_{n}\left\|x_{n}-x^{*}\right\|+\sigma_{n}\left\|x_{n}-x^{*}\right\|+\eta_{n}\left\|\alpha_{n}\left(f\left(y_{n}\right)-x^{*}\right)+\left(1-\alpha_{n}\right)\left(\widetilde{G}^{x} x_{n}-x^{*}\right)\right\| \\
& \leq\left(1-\eta_{n}\right)\left\|x_{n}-x^{*}\right\|+\eta_{n}\left[\alpha_{n}\left\|f\left(y_{n}\right)-x^{*}\right\|+\left(1-\alpha_{n}\right)\left\|\widetilde{G}^{x} x_{n}-x^{*}\right\|\right] \\
& \leq\left(1-\eta_{n}\right)\left\|x_{n}-x^{*}\right\|+\eta_{n}\left[\alpha_{n}\left\|f\left(y_{n}\right)-f\left(y^{*}\right)\right\|+\alpha_{n}\left\|f\left(y^{*}\right)-x^{*}\right\|+\left(1-\alpha_{n}\right)\left\|x_{n}-x^{*}\right\|\right] \\
& \leq\left(1-\eta_{n}\right)\left\|x_{n}-x^{*}\right\|+\eta_{n}\left[\alpha_{n} \rho_{f}\left\|y_{n}-y^{*}\right\|+\alpha_{n}\left\|f\left(y^{*}\right)-x^{*}\right\|+\left(1-\alpha_{n}\right)\left\|x_{n}-x^{*}\right\|\right] \\
& \leq\left(1-\eta_{n}\right)\left\|x_{n}-x^{*}\right\|+\eta_{n} \alpha_{n} \rho\left\|y_{n}-y^{*}\right\|+\eta_{n} \alpha_{n}\left\|f\left(y^{*}\right)-x^{*}\right\|+\eta_{n}\left(1-\alpha_{n}\right)\left\|x_{n}-x^{*}\right\| \\
& =\left(1-\eta_{n} \alpha_{n}\right)\left\|x_{n}-x^{*}\right\|+\eta_{n} \alpha_{n} \rho\left\|y_{n}-y^{*}\right\|+\eta_{n} \alpha_{n}\left\|f\left(y^{*}\right)-x^{*}\right\| .
\end{aligned}
$$

Similarly, from definition of $y_{n}$, we have

$$
\left\|y_{n+1}-y^{*}\right\| \leq\left(1-\eta_{n} \alpha_{n}\right)\left\|y_{n}-y^{*}\right\|+\eta_{n} \alpha_{n} \rho\left\|x_{n}-x^{*}\right\|+\eta_{n} \alpha_{n}\left\|g\left(x^{*}\right)-y^{*}\right\| .
$$

Hence, from (23) and (24), we obtain

$$
\begin{aligned}
\left\|x_{n+1}-x^{*}\right\|+\left\|y_{n+1}-y^{*}\right\| \leq & \left(1-\eta_{n} \alpha_{n}\right)\left(\left\|x_{n}-x^{*}\right\|+\left\|y_{n}-y^{*}\right\|\right)+\eta_{n} \alpha_{n} \rho\left(\left\|x_{n}-x^{*}\right\|+\left\|y_{n}-y^{*}\right\|\right) \\
& +\eta_{n} \alpha_{n}\left(\left\|f\left(y^{*}\right)-x^{*}\right\|+\left\|g\left(x^{*}\right)-y^{*}\right\|\right) \\
= & \left(1-(1-\rho) \eta_{n} \alpha_{n}\right)\left(\left\|x_{n}-x^{*}\right\|+\left\|y_{n}-y^{*}\right\|\right)+\eta_{n} \alpha_{n}\left(\left\|f\left(y^{*}\right)-x^{*}\right\|+\left\|g\left(x^{*}\right)-y^{*}\right\|\right) .
\end{aligned}
$$

By induction, we have 


$$
\left\|x_{n}-x^{*}\right\|+\left\|y_{n}-y^{*}\right\| \leq \max \left\{\left\|x_{1}-x^{*}\right\|+\left\|y_{1}-y^{*}\right\|, \frac{\left\|f\left(y^{*}\right)-x^{*}\right\|+\left\|g\left(x^{*}\right)-y^{*}\right\|}{1-\rho}\right\},
$$

for every $n \in \mathbb{N}$. Thus, $\left\{x_{n}\right\}$ and $\left\{y_{n}\right\}$ are bounded.

Step 2. We will show that $\lim _{n \longrightarrow \infty}\left\|x_{n+1}-x_{n}\right\|=$ $\lim _{n \longrightarrow \infty}\left\|y_{n+1}-y_{n}\right\|=0$. Put $u_{n}=P_{C}\left(I-\mu_{n}^{x} \sum_{i=1}^{N} a_{i}^{x} B_{i}^{x}\right) x_{n}$,
$v_{n}=P_{C}\left(I-\mu_{n}^{y} \sum_{i=1}^{N} a_{i}^{y} B_{i}^{y}\right) y_{n}, z_{n}=\alpha_{n} f\left(y_{n}\right)+\left(1-\alpha_{n}\right) \widetilde{G}^{x} x_{n}$, and $w_{n}=\alpha_{n} f\left(x_{n}\right)+\left(1-\alpha_{n}\right) \widetilde{G}^{y} y_{n}$, for all $n \geq 1$. From Lemma 6 , we have

$$
\begin{aligned}
\left\|z_{n}-z_{n-1}\right\| & =\left\|\left(\alpha_{n} f\left(y_{n}\right)+\left(1-\alpha_{n}\right) \widetilde{G}^{x} x_{n}\right)-\left(\alpha_{n-1} f\left(y_{n-1}\right)+\left(1-\alpha_{n-1}\right) \widetilde{G}^{x} x_{n-1}\right)\right\|, \\
& \leq \alpha_{n}\left\|f\left(y_{n}\right)-f\left(y_{n-1}\right)\right\|+\left(1-\alpha_{n}\right)\left\|\widetilde{G}^{x} x_{n}-\widetilde{G}^{x} x_{n-1}\right\|+\left|\alpha_{n}-\alpha_{n-1}\right|\left\|f\left(y_{n-1}\right)\right\|+\mid \alpha_{n}-\alpha_{n-1}\left\|\widetilde{G}^{x} x_{n-1}\right\| \\
& \leq \alpha_{n} \rho_{f}\left\|y_{n}-y_{n-1}\right\|+\left(1-\alpha_{n}\right)\left\|x_{n}-x_{n-1}\right\|+\left|\alpha_{n}-\alpha_{n-1}\right|\left\|f\left(y_{n-1}\right)\right\|+\left|\alpha_{n}-\alpha_{n-1}\right|\left\|\widetilde{G}^{x} x_{n-1}\right\| \\
& \leq \alpha_{n} \rho\left\|y_{n}-y_{n-1}\right\|+\left(1-\alpha_{n}\right)\left\|x_{n}-x_{n-1}\right\|+\left|\alpha_{n}-\alpha_{n-1}\right|\left(\left\|f\left(y_{n-1}\right)\right\|+\left\|\widetilde{G}^{x} x_{n-1}\right\|\right) .
\end{aligned}
$$

By applying Lemma 3, we get that

$$
\begin{aligned}
\left\|u_{n}-u_{n-1}\right\| & =\left\|P_{C}\left(I-\mu_{n}^{x} \sum_{i=1}^{N} a_{i}^{x} B_{i}^{x}\right) x_{n}-P_{C}\left(I-\mu_{n-1}^{x} \sum_{i=1}^{N} a_{i}^{x} B_{i}^{x}\right) x_{n-1}\right\|, \\
& \leq\left\|x_{n}-x_{n-1}\right\|+\left\|P_{C}\left(I-\mu_{n}^{x} \sum_{i=1}^{N} a_{i}^{x} B_{i}^{x}\right) x_{n-1}-P_{C}\left(I-\mu_{n-1}^{x} \sum_{i=1}^{N} a_{i}^{x} B_{i}^{x}\right) x_{n-1}\right\| a_{i}^{x}\left\|B_{i}^{x} x_{n-1}\right\| . \\
& \leq\left\|x_{n}-x_{n-1}\right\|+\left|\mu_{n}^{x}-\mu_{n-1}^{x}\right| \sum_{i=1}^{N}
\end{aligned}
$$

From the definition of $\left\{x_{n}\right\},(27)$, and (28), we have

$$
\begin{aligned}
\left\|x_{n+1}-x_{n}\right\|= & \left\|\left(\delta_{n} x_{n}+\sigma_{n} u_{n}+\eta_{n} z_{n}\right)-\left(\delta_{n-1} x_{n-1}+\sigma_{n-1} u_{n-1}+\eta_{n-1} z_{n-1}\right)\right\|, \\
\leq & \delta_{n}\left\|x_{n}-x_{n-1}\right\|+\left|\delta_{n}-\delta_{n-1}\right|\left\|x_{n-1}\right\|+\sigma_{n}\left\|u_{n}-u_{n-1}\right\|+\left|\sigma_{n}-\sigma_{n-1}\right|\left\|u_{n-1}\right\|+\eta_{n}\left\|z_{n}-z_{n-1}\right\|+\left|\eta_{n}-\eta_{n-1}\right|\left\|z_{n-1}\right\| \\
\leq & \delta_{n}\left\|x_{n}-x_{n-1}\right\|+\left|\delta_{n}-\delta_{n-1}\right|\left\|x_{n-1}\right\|+\sigma_{n}\left[\left\|x_{n}-x_{n-1}\right\|+\left|\mu_{n}^{x}-\mu_{n-1}^{x}\right| \sum_{i=1}^{N} a_{i}^{x}\left\|B_{i}^{x} x_{n-1}\right\|\right]+\left|\sigma_{n}-\sigma_{n-1}\right|\left\|u_{n-1}\right\| \\
& +\eta_{n}\left[\alpha_{n} \rho\left\|y_{n}-y_{n-1}\right\|+\left(1-\alpha_{n}\right)\left\|x_{n}-x_{n-1}\right\|+\left|\alpha_{n}-\alpha_{n-1}\right|\left(\left\|f\left(y_{n-1}\right)\right\|+\left\|\widetilde{G}^{x} x_{n-1}\right\|\right)\right]+\left|\eta_{n}-\eta_{n-1}\right|\left\|z_{n-1}\right\| \\
= & \left(1-\eta_{n} \alpha_{n}\right)\left\|x_{n}-x_{n-1}\right\|+\eta_{n} \alpha_{n} \rho\left\|y_{n}-y_{n-1}\right\|+\sigma_{n}\left|\mu_{n}^{x}-\mu_{n-1}^{x}\right| \sum_{i=1}^{N} a_{i}^{x}\left\|B_{i}^{x} x_{n-1}\right\| \\
& +\left|\delta_{n}-\delta_{n-1}\right|\left\|x_{n-1}\right\|+\left|\sigma_{n}-\sigma_{n-1}\right|\left\|u_{n-1}\right\|+\eta_{n}\left|\alpha_{n}-\alpha_{n-1}\right|\left(\left\|f\left(y_{n-1}\right)\right\|+\left\|\widetilde{G}^{x} x_{n-1}\right\|\right)+\left|\eta_{n}-\eta_{n-1}\right|\left\|z_{n-1}\right\| .
\end{aligned}
$$

By the same argument as in (27) and (29), we also have 


$$
\begin{aligned}
\left\|w_{n}-w_{n-1}\right\| \leq & \alpha_{n} \rho\left\|x_{n}-x_{n-1}\right\|+\left(1-\alpha_{n}\right)\left\|y_{n}-y_{n-1}\right\|+\left|\alpha_{n}-\alpha_{n-1}\right|\left(\left\|g\left(x_{n-1}\right)\right\|+\left\|\widetilde{G}^{y} y_{n-1}\right\|\right) . \\
\left\|y_{n+1}-y_{n}\right\| \leq & \left(1-\eta_{n} \alpha_{n}\right)\left\|y_{n}-y_{n-1}\right\|+\eta_{n} \alpha_{n} \rho\left\|x_{n}-x_{n-1}\right\|+\sigma_{n}\left|\mu_{n}^{y}-\mu_{n-1}^{y}\right| \sum_{i=1}^{N} a_{i}^{y}\left\|B_{i}^{y} y_{n-1}\right\| \\
& +\left|\delta_{n}-\delta_{n-1}\right|\left\|y_{n-1}\right\|+\left|\sigma_{n}-\sigma_{n-1}\right|\left\|v_{n-1}\right\|+\eta_{n}\left|\alpha_{n}-\alpha_{n-1}\right|\left(\left\|g\left(x_{n-1}\right)\right\|+\left\|\widetilde{G}^{y} y_{n-1}\right\|\right)+\left|\eta_{n}-\eta_{n-1}\right|\left\|w_{n-1}\right\| .
\end{aligned}
$$

From (29) and (31), we obtain that

$$
\begin{aligned}
\left\|x_{n+1}-x_{n}\right\|+\left\|y_{n+1}-y_{n}\right\| \leq & \left(1-(1-\rho) \eta_{n} \alpha_{n}\right)\left(\left\|x_{n}-x_{n-1}\right\|+\left\|y_{n}-y_{n-1}\right\|\right) \\
& +\sigma_{n}\left|\mu_{n}^{x}-\mu_{n-1}^{x}\right|\left(\sum_{i=1}^{N} a_{i}^{x}\left\|B_{i}^{x} x_{n-1}\right\|+\sum_{i=1}^{N} a_{i}^{y}\left\|B_{i}^{y} y_{n-1}\right\|\right)+\left|\delta_{n}-\delta_{n-1} \|\left(\left\|x_{n-1}\right\|+\left\|y_{n-1}\right\|\right)+\right| \sigma_{n}-\sigma_{n-1} \mid\left(\left\|u_{n-1}\right\|+\left\|v_{n-1}\right\|\right) \\
& +\eta_{n}\left|\alpha_{n}-\alpha_{n-1}\right|\left(\left\|f\left(y_{n-1}\right)\right\|+\left\|g\left(x_{n-1}\right)\right\|+\left\|\widetilde{G}^{x} x_{n-1}\right\|+\left\|\widetilde{G}^{y} y_{n-1}\right\|\right)+\left|\eta_{n}-\eta_{n-1}\right|\left(\left\|z_{n-1}\right\|+\left\|w_{n-1}\right\|\right) \\
\leq & \left(1-(1-\rho) \bar{a} \alpha_{n}\right)\left(\left\|x_{n}-x_{n-1}\right\|+\left\|y_{n}-y_{n-1}\right\|\right)+\bar{b}\left|\mu_{n}^{x}-\mu_{n-1}^{x}\right|\left(\sum_{i=1}^{N} a_{i}^{x}\left\|B_{i}^{x} x_{n-1}\right\|+\sum_{i=1}^{N} a_{i}^{y}\left\|B_{i}^{y} y_{n-1}\right\|\right) \\
& +\left|\delta_{n}-\delta_{n-1} \|\left(\left\|x_{n-1}\right\|+\left\|y_{n-1}\right\|\right)+\right| \sigma_{n}-\sigma_{n-1} \mid\left(\left\|u_{n-1}\right\|+\left\|v_{n-1}\right\|\right) \\
& +\bar{b}\left|\alpha_{n}-\alpha_{n-1}\right|\left(\left\|f\left(y_{n-1}\right)\right\|+\left\|g\left(x_{n-1}\right)\right\|+\left\|\widetilde{G}^{x} x_{n-1}\right\|+\left\|\widetilde{G}^{y} y_{n-1}\right\|\right)+\left|\eta_{n}-\eta_{n-1}\right|\left(\left\|z_{n-1}\right\|+\left\|w_{n-1}\right\|\right) .
\end{aligned}
$$

From (32), conditions (1), (2), and (5), and Lemma 8, we obtain that

$$
\begin{aligned}
& \lim _{n \longrightarrow \infty}\left\|x_{n+1}-x_{n}\right\|=0, \\
& \lim _{n \longrightarrow \infty}\left\|y_{n+1}-y_{n}\right\|=0 .
\end{aligned}
$$

$$
\begin{aligned}
\left\|x_{n+1}-x^{*}\right\|^{2} & =\left\|\delta_{n}\left(x_{n}-x^{*}\right)+\sigma_{n}\left(P_{C}\left(I-\mu_{n}^{x} \sum_{i=1}^{N} a_{i}^{x} B_{i}^{x}\right) x_{n}-x^{*}\right)+\eta_{n}\left(z_{n}-x^{*}\right)\right\|^{2}, \\
& \leq \delta_{n}\left\|x_{n}-x^{*}\right\|^{2}+\sigma_{n}\left\|P_{C}\left(I-\mu_{n}^{x} \sum_{i=1}^{N} a_{i}^{x} B_{i}^{x}\right) x_{n}-x^{*}\right\|^{2}-\delta_{n} \sigma_{n}\left\|x_{n}-P_{C}\left(I-\mu_{n}^{x} \sum_{i=1}^{N} a_{i}^{x} B_{i}^{x}\right) x_{n}\right\|^{2}+\eta_{n}\left\|z_{n}-x^{*}\right\|^{2} \\
& \leq\left(1-\eta_{n}\right)\left\|x_{n}-x^{*}\right\|^{2}-\delta_{n} \sigma_{n}\left\|x_{n}-P_{C}\left(I-\mu_{n}^{x} \sum_{i=1}^{N} a_{i}^{x} B_{i}^{x}\right) x_{n}\right\|^{2}+\eta_{n}\left\|\alpha_{n}\left(f\left(y_{n}-\widetilde{G}^{x} x_{n}\right)+\left(\widetilde{G}^{x} x_{n}-x^{*}\right)\right)\right\|^{2} \\
& \leq\left(1-\eta_{n}\right)\left\|x_{n}-x^{*}\right\|^{2}-\delta_{n} \sigma_{n}\left\|x_{n}-P_{C}\left(I-\mu_{n}^{x} \sum_{i=1}^{N} a_{i}^{x} B_{i}^{x}\right) x_{n}\right\|^{2}+\eta_{n}\left[\left\|\widetilde{G}^{x} x_{n}-x^{*}\right\|^{2}+2 \alpha_{n}\left\langle f\left(y_{n}\right)-\widetilde{G}^{x} x_{n}, z_{n}-x^{*}\right\rangle\right] \\
& \leq\left\|x_{n}-x^{*}\right\|^{2}-\delta_{n} \sigma_{n}\left\|x_{n}-P_{C}\left(I-\mu_{n}^{x} \sum_{i=1}^{N} a_{i}^{x} B_{i}^{x}\right) x_{n}\right\|^{2}+2 \eta_{n} \alpha_{n}\left\|f\left(y_{n}\right)-\widetilde{G}^{x} x_{n}\right\|\left\|z_{n}-x^{*}\right\|,
\end{aligned}
$$

$$
\begin{aligned}
\delta_{n} \sigma_{n}\left\|x_{n}-P_{C}\left(I-\mu_{n}^{x} \sum_{i=1}^{N} a_{i}^{x} B_{i}^{x}\right) x_{n}\right\|^{2} & \leq\left\|x_{n}-x^{*}\right\|^{2}-\left\|x_{n+1}-x^{*}\right\|^{2}+2 \eta_{n} \alpha_{n}\left\|f\left(y_{n}\right)-\widetilde{G}^{x} x_{n}\right\|\left\|z_{n}-x^{*}\right\|, \\
& \leq\left\|x_{n}-x_{n+1}\right\|\left[\left\|x_{n}-x^{*}\right\|+\left\|x_{n+1}-x^{*}\right\|\right]+2 \eta_{n} \alpha_{n}\left\|f\left(y_{n}\right)-\widetilde{G}^{x} x_{n}\right\|\left\|z_{n}-x^{*}\right\| .
\end{aligned}
$$


Then, we have

$\left\|x_{n}-P_{C}\left(I-\mu_{n}^{x} \sum_{i=1}^{N} a_{i}^{x} B_{i}^{x}\right) x_{n}\right\| \longrightarrow 0 \quad$ as $n \longrightarrow \infty$.

Observe that

$x_{n+1}-x_{n}=\sigma_{n}\left(P_{C}\left(I-\mu_{n}^{x} \sum_{i=1}^{N} a_{i}^{x} B_{i}^{x}\right) x_{n}-x_{n}\right)+\eta_{n}\left(z_{n}-x_{n}\right)$.
From (33) and (37), we have

$$
\left\|z_{n}-x_{n}\right\| \longrightarrow 0 \text { as } n \longrightarrow \infty \text {. }
$$

By the same argument as above, we also have that

$$
\left\|w_{n}-x_{n}\right\| \longrightarrow 0 \text { as } n \longrightarrow \infty \text {. }
$$

Note that

$$
\begin{aligned}
\left\|z_{n}-P_{C}\left(I-\mu_{n}^{x} \sum_{i=1}^{N} a_{i}^{x} B_{i}^{x}\right) z_{n}\right\| \leq & \left\|z_{n}-x_{n}\right\|+\left\|x_{n}-P_{C}\left(I-\mu_{n}^{x} \sum_{i=1}^{N} a_{i}^{x} B_{i}^{x}\right) x_{n}\right\| \\
& +\left\|P_{C}\left(I-\mu_{n}^{x} \sum_{i=1}^{N} a_{i}^{x} B_{i}^{x}\right) x_{n}-P_{C}\left(I-\mu_{n}^{x} \sum_{i=1}^{N} a_{i}^{x} B_{i}^{x}\right) z_{n}\right\|, \\
\leq & \left\|z_{n}-x_{n}\right\|+\left\|x_{n}-P_{C}\left(I-\mu_{n}^{x} \sum_{i=1}^{N} a_{i}^{x} B_{i}^{x}\right) x_{n}\right\|+\left\|x_{n}-z_{n}\right\| \\
& =2\left\|z_{n}-x_{n}\right\|+\left\|x_{n}-P_{C}\left(I-\mu_{n}^{x} \sum_{i=1}^{N} a_{i}^{x} B_{i}^{x}\right) x_{n}\right\|,
\end{aligned}
$$

By (37) and (39), we get that

$\left\|z_{n}-P_{C}\left(I-\mu_{n}^{x} \sum_{i=1}^{N} a_{i}^{x} B_{i}^{x}\right) z_{n}\right\| \longrightarrow 0 \quad$ as $n \longrightarrow \infty$.

By the same argument as (41), we also obtain

$\left\|w_{n}-P_{C}\left(I-\mu_{n}^{y} \sum_{i=1}^{N} a_{i}^{y} B_{i}^{y}\right) w_{n}\right\| \longrightarrow 0 \quad$ as $n \longrightarrow \infty$.
Consider

$$
\left\|x_{n+1}-z_{n}\right\| \leq\left\|x_{n+1}-x_{n}\right\|+\left\|x_{n}-z_{n}\right\| .
$$

By (33) and (39), we get that

$$
\left\|x_{n+1}-z_{n}\right\| \longrightarrow 0 \text { as } n \longrightarrow \infty \text {. }
$$

However,

$$
\begin{aligned}
\left\|x_{n}-\widetilde{G}^{x} x_{n}\right\| & \leq\left\|x_{n}-x_{n+1}\right\|+\left\|x_{n+1}-z_{n}\right\|+\left\|z_{n}-\widetilde{G}^{x} x_{n}\right\|, \\
& =\left\|x_{n}-x_{n+1}\right\|+\left\|x_{n+1}-z_{n}\right\|+\left\|\alpha_{n} f\left(y_{n}\right)+\left(1-\alpha_{n}\right) \widetilde{G}^{x} x_{n}-\widetilde{G}^{x} x_{n}\right\| \\
& =\left\|x_{n}-x_{n+1}\right\|+\left\|x_{n+1}-z_{n}\right\|+\alpha_{n}\left\|f\left(y_{n}\right)-\widetilde{G}^{x} x_{n}\right\|,
\end{aligned}
$$

It follows from (33) and (45) that

$$
\left\|x_{n}-\widetilde{G}^{x} x_{n}\right\| \longrightarrow 0 \text { as } n \longrightarrow \infty \text {. }
$$

\section{Consider}

$$
\begin{aligned}
\left\|z_{n}-\widetilde{G}^{x} z_{n}\right\| & \leq\left\|z_{n}-x_{n}\right\|+\left\|x_{n}-\widetilde{G}^{x} x_{n}\right\|+\left\|\widetilde{G}^{x} x_{n}-\widetilde{G}^{x} z_{n}\right\|, \\
& \leq 2\left\|z_{n}-x_{n}\right\|+\left\|x_{n}-\widetilde{G}^{x} x_{n}\right\| .
\end{aligned}
$$

From (39) and (47), we obtain

$$
\left\|z_{n}-\widetilde{G}^{x} z_{n}\right\| \longrightarrow 0 \text { as } n \longrightarrow \infty \text {. }
$$

Applying the same method as (48), we also have

$$
\left\|w_{n}-\widetilde{G}^{y} w_{n}\right\| \longrightarrow 0 \text { as } n \longrightarrow \infty \text {. }
$$

Step 4. We will show that $\limsup _{n \rightarrow \infty}\langle f$ $\left.(\widetilde{y})-\tilde{x}, z_{n}-\tilde{x}\right\rangle \leq 0$ and $\lim \sup _{n \rightarrow \infty}\left\langle g(\tilde{x})-\tilde{y}, z_{n}-\tilde{y}\right\rangle \leq 0$, where $\tilde{x}=P_{\mathscr{F}^{x}} f(\tilde{y})$ and $\tilde{y}=P_{\mathscr{F}^{y}} f(\tilde{x})$. First, we take a subsequence $\left\{z_{n_{k}}\right\}$ of $\left\{z_{n}\right\}$ such that $\limsup _{n \longrightarrow \infty}\left\langle f(\tilde{y})-\tilde{x}, z_{n}-\tilde{x}\right\rangle=\lim _{k \longrightarrow \infty}\left\langle f(\tilde{y})-\tilde{x}, z_{n_{k}}-\tilde{x}\right\rangle$. 
Since $\left\{x_{n}\right\}$ is bounded, there exists a subsequence $x_{n_{k}}$ of $\left\{x_{n}\right\}$ such that $x_{n_{k}} \rightarrow q_{1}$ as $k \longrightarrow \infty$. From (39), we get that $z_{n_{k}} \rightarrow q_{1}$. Next, we need to show that $q_{1} \in \mathscr{F}^{x}=\Omega^{x} \cap\left(\cap_{i=1}^{N} V I\left(\underset{\widetilde{G}_{x}}{C} B_{i}^{x}\right)\right)$. Assume that $q_{1} \notin \Omega^{x}$. By Lemma 7, we get that $q_{1} \neq \widetilde{G}^{x} q_{1}$. Applying Opial's condition and (49), we get that

$$
\begin{aligned}
\liminf _{k \rightarrow \infty}\left\|z_{n_{k}}-q_{1}\right\| & <\liminf _{k \longrightarrow \infty}\left\|z_{n_{k}}-\widetilde{G}^{x} q_{1}\right\|, \\
& \leq \liminf _{n \longrightarrow \infty}\left\|z_{n_{k}}-\widetilde{G}^{x} z_{n_{k}}\right\|+\liminf \inf _{k \rightarrow \infty}\left\|\widetilde{G}^{x} z_{n_{k}}-\widetilde{G}^{x} q_{1}\right\| \\
& \leq \liminf _{k \longrightarrow \infty}\left\|z_{n_{k}}-q_{1}\right\| .
\end{aligned}
$$

This is a contradiction. Thus, $q_{1} \in \Omega^{x}$.

Assume that $q_{1} \notin \cap_{i=1}^{N} V I\left(C, B_{i}^{x}\right)$. Then, from Lemma 3 and Lemma 2, we have $q_{1} \notin F\left(P_{C}\left(I-\mu_{n}^{x} \sum_{i=1}^{N} a_{i} B_{i}^{x}\right)\right)$. From Opial's condition and (42), we obtain

$$
\begin{aligned}
\liminf _{k \rightarrow \infty}\left\|z_{n_{k}}-q_{1}\right\| & <\liminf _{k \rightarrow \infty}\left\|z_{n_{k}}-P_{C}\left(I-\mu_{n_{k}}^{x} \sum_{i=1}^{N} a_{i}^{x} B_{i}^{x}\right) q_{1}\right\| \\
& \leq \liminf _{k \rightarrow \infty}\left\|z_{n_{k}}-P_{C}\left(I-\mu_{n_{k}}^{x} \sum_{i=1}^{N} a_{i}^{x} B_{i}^{x}\right) z_{n_{k}}\right\|+\liminf _{k \rightarrow \infty}\left\|P_{C}\left(I-\mu_{n_{k}}^{x} \sum_{i=1}^{N} a_{i}^{x} B_{i}^{x}\right) z_{n_{k}}-P_{C}\left(I-\mu_{n_{k}} \sum_{i=1}^{N} a_{i}^{x} B_{i}^{x}\right) q_{1}\right\| \\
& \leq \liminf _{k \rightarrow \infty}\left\|z_{n_{k}}-q_{1}\right\| .
\end{aligned}
$$

This is a contradiction. Thus, $q_{1} \in \cap_{i=1}^{N} V I\left(C, B_{i}^{x}\right)$, and so,

$$
q_{1} \in \mathscr{F}^{x}=\Omega^{x} \cap\left(\bigcap_{i=1}^{N} V I\left(C, B_{i}^{x}\right)\right) .
$$

However, $z_{n_{k}} \rightarrow q_{1}$. From (54) and Lemma 1, we can derive that

$$
\begin{aligned}
\underset{n \longrightarrow \infty}{\limsup }\left\langle f(\tilde{y})-\tilde{x}, z_{n}-\tilde{x}\right\rangle & =\lim _{k \longrightarrow \infty}\left\langle f(\tilde{y})-\tilde{x}, z_{n_{k}}-\tilde{x}\right\rangle, \\
& =\left\langle f(\tilde{y})-\tilde{x}, q_{1}-\tilde{x}\right\rangle \\
& \leq 0 .
\end{aligned}
$$

By the same method as (55), we also obtain that

$$
\limsup _{n \longrightarrow \infty}\left\langle g(\tilde{x})-\tilde{y}, z_{n}-\tilde{y}\right\rangle \leq 0 \text {. }
$$

Step 5. Finally, we show that the sequences $\left\{x_{n}\right\}$ and $\left\{y_{n}\right\}$ converges strongly to $\tilde{x}=P_{\mathscr{F}^{x}} f(\tilde{y})$ and $\tilde{y}=P_{\mathscr{F}^{y}} f(\tilde{x})$, respectively. From the definition of $z_{n}$, we have

$$
\begin{aligned}
\left\|z_{n}-\tilde{x}\right\|^{2} & =\left\langle\alpha_{n}\left(f\left(y_{n}\right)-\tilde{x}\right)+\left(1-\alpha_{n}\right)\left(\tilde{G}^{x} x_{n}-\tilde{x}\right), z_{n}-\tilde{x}\right\rangle, \\
& =\alpha_{n}\left\langle f\left(y_{n}\right)-\tilde{x}, z_{n}-\tilde{x}\right\rangle+\left(1-\alpha_{n}\right)\left\langle\tilde{G}^{x} x_{n}-\tilde{x}, z_{n}-\tilde{x}\right\rangle \\
& \leq \alpha_{n}\left\langle f\left(y_{n}\right)-f(\tilde{y}), z_{n}-\tilde{x}\right\rangle+\alpha_{n}\left\langle f(\tilde{y})-\tilde{x}, z_{n}-\tilde{x}\right\rangle+\left(1-\alpha_{n}\right)\left\|\widetilde{G}^{x} x_{n}-\tilde{x}\right\|\left\|z_{n}-\tilde{x}\right\| \\
& \leq \alpha_{n} \rho\left\|y_{n}-\tilde{y}\right\|\left\|z_{n}-\tilde{x}\right\|+\alpha_{n}\left\langle f(\tilde{y})-\tilde{x}, z_{n}-\tilde{x}\right\rangle+\left(1-\alpha_{n}\right)\left\|x_{n}-\tilde{x}\right\|\left\|z_{n}-\tilde{x}\right\| \\
& \leq \frac{\alpha_{n} \rho}{2}\left[\left\|y_{n}-\tilde{y}\right\|^{2}+\left\|z_{n}-\tilde{x}\right\|^{2}\right]+\alpha_{n}\left\langle f(\tilde{y})-\tilde{x}, z_{n}-\tilde{x}\right\rangle+\frac{\left(1-\alpha_{n}\right)}{2}\left[\left\|x_{n}-\tilde{x}\right\|^{2}+\left\|z_{n}-\tilde{x}\right\|^{2}\right],
\end{aligned}
$$

which implies that 


$$
\left\|z_{n}-\tilde{x}\right\|^{2} \leq \frac{\alpha_{n} \rho}{1+\alpha_{n}(1-\rho)}\left\|y_{n}-\tilde{y}\right\|^{2}+\frac{\left(1-\alpha_{n}\right)}{1+\alpha_{n}(1-\rho)}\left\|x_{n}-\tilde{x}\right\|^{2}+\frac{2 \alpha_{n}}{1+\alpha_{n}(1-\rho)}\left\langle f(\tilde{y})-\tilde{x}, z_{n}-\tilde{x}\right\rangle .
$$

From the definition of $\left\{x_{n}\right\}$ and (58), we get

$$
\begin{aligned}
\left\|x_{n+1}-\tilde{x}\right\|^{2} & \leq \delta_{n}\left\|x_{n}-\tilde{x}\right\|^{2}+\sigma_{n}\left\|P_{C}\left(I-\mu_{n}^{x} \sum_{i=1}^{N} a_{i}^{x} B_{i}^{x}\right) x_{n}-\tilde{x}\right\|^{2}+\eta_{n}\left\|z_{n}-\tilde{x}\right\|^{2}, \\
& \leq\left(1-\eta_{n}\right)\left\|x_{n}-\tilde{x}\right\|^{2}+\frac{\alpha_{n} \eta_{n} \rho}{1+\alpha_{n}(1-\rho)}\left\|y_{n}-\tilde{y}\right\|^{2}+\frac{\left(1-\alpha_{n}\right) \eta_{n}}{1+\alpha_{n}(1-\rho)}\left\|x_{n}-\tilde{x}\right\|^{2}+\frac{2 \alpha_{n} \eta_{n}}{1+\alpha_{n}(1-\rho)}\left\langle f(\tilde{y})-\tilde{x}, z_{n}-\tilde{x}\right\rangle \\
& =\left(1-\frac{\alpha_{n} \eta_{n}(2-\rho)}{1+\alpha_{n}(1-\rho)}\right)\left\|x_{n}-\tilde{x}\right\|^{2}+\frac{\alpha_{n} \eta_{n} \rho}{1+\alpha_{n}(1-\rho)}\left\|y_{n}-\tilde{y}\right\|^{2}+\frac{2 \alpha_{n} \eta_{n}}{1+\alpha_{n}(1-\rho)}\left\langle f(\tilde{y})-\tilde{x}, z_{n}-\tilde{x}\right\rangle .
\end{aligned}
$$

Applying the same argument as in (58) and (59), we get

$$
\left\|y_{n+1}-\tilde{y}\right\|^{2} \leq\left(1-\frac{\alpha_{n} \eta_{n}(2-\rho)}{1+\alpha_{n}(1-\rho)}\right)\left\|y_{n}-\tilde{y}\right\|^{2}+\frac{\alpha_{n} \eta_{n} \rho}{1+\alpha_{n}(1-\rho)}\left\|x_{n}-\tilde{x}\right\|^{2}+\frac{2 \alpha_{n} \eta_{n}}{1+\alpha_{n}(1-\rho)}\left\langle g(\tilde{x})-\tilde{y}, z_{n}-\tilde{y}\right\rangle
$$

From (58) and (59), we have

$$
\begin{aligned}
\left\|x_{n+1}-\tilde{x}\right\|^{2}+\left\|y_{n+1}-\tilde{y}\right\|^{2} \leq & \left(1-\frac{\alpha_{n} \eta_{n}(2-\rho)}{1+\alpha_{n}(1-\rho)}\right)\left[\left\|x_{n}-\tilde{x}\right\|^{2}+\left\|y_{n}-\tilde{y}\right\|^{2}\right] \\
& +\frac{\alpha_{n} \eta_{n} \rho}{1+\alpha_{n}(1-\rho)}\left[\left\|x_{n}-\tilde{x}\right\|^{2}+\left\|y_{n}-\tilde{y}\right\|^{2}\right]+\frac{2 \alpha_{n} \eta_{n}}{1+\alpha_{n}(1-\rho)}\left[\left\langle f(\tilde{y})-\tilde{x}, z_{n}-\tilde{x}\right\rangle+\left\langle g(\tilde{x})-\tilde{y}, z_{n}-\tilde{y}\right\rangle\right], \\
\leq & \left(1-\frac{2 \alpha_{n} \eta_{n}(1-\rho)}{1+\alpha_{n}(1-\rho)}\right)\left[\left\|x_{n}-\tilde{x}\right\|^{2}+\left\|y_{n}-\tilde{y}\right\|^{2}\right]+\frac{2 \alpha_{n} \eta_{n}}{1+\alpha_{n}(1-\rho)}\left[\left\langle f(\tilde{y})-\tilde{x}, z_{n}-\tilde{x}\right\rangle+\left\langle g(\tilde{x})-\tilde{y}, z_{n}-\tilde{y}\right\rangle\right] .
\end{aligned}
$$

According to condition (2) and (4), (61), and Lemma 8, we can conclude that $\left\{x_{n}\right\}$ and $\left\{y_{n}\right\}$ converge strongly to $\tilde{x}=P_{\mathscr{F}^{x}} f(\tilde{y})$ and $\tilde{y}=P_{\mathscr{F}^{y}} g(\tilde{x})$, respectively. Furthermore, from (39) and (40), we get that $\left\{z_{n}\right\}$ and $\left\{w_{n}\right\}$ converge strongly to $\tilde{x}=P_{\mathscr{F}^{x}} f(\tilde{y})$ and $\tilde{y}=P_{\mathscr{F}^{y}} g(\tilde{x})$, respectively. This completes the proof.

One of the great special cases of the SMVIP is the split variational inclusion problem that has a wide variety of application backgrounds, such as split minimization problems and split feasibility problems.
If we set $A_{i}^{x}=0$ and $A_{i}^{y}=0$ in Theorem 2 , for all $i=1,2$, then we get the strong convergence theorem for the split variational inclusion problem and the finite families of the variational inequality problems as follows:

Corollary 1. Let $H_{1}$ and $H_{2}$ be Hilbert spaces, and let $C$ be a nonempty closed convex subset of $H_{1}$. Let $T: H_{1} \longrightarrow H_{2}$ be a bounded linear operator, and let $f, g: H_{1} \longrightarrow H_{1}$ be $\rho_{f}, \rho_{g}$-contraction mappings with $\rho=\max \left\{\rho_{f}, \rho_{g}\right\}$. For every $i=1,2$, let $M_{i}^{x}, M_{i}^{y}: H_{i} \longrightarrow 2^{H_{i}}$ be multivalued maximal monotone mappings. For $i=1,2, \ldots, N$, let $B_{i}^{x}, B_{i}^{y}$ : 
$H_{1} \longrightarrow H_{1}$ be $\beta_{i}^{x}, \beta_{i}^{y}$-inverse strongly monotone with $\bar{\beta}_{x}=$ $\min _{i=1,2, \ldots, N}\left\{\beta_{i}^{x}\right\}$ and $\bar{\beta}_{y}=\min _{i=1,2, \ldots, N}\left\{\beta_{i}^{y}\right\}$. Let $\mathcal{S}^{x}=\left\{x^{*} \in\right.$ $\left.H_{1}: 0 \in M_{1}^{x} x^{*}, \tilde{x}=T x^{*} \in H_{2}: 0 \in M_{2}^{x} \widetilde{x}\right\}$ and $\mathcal{S}^{y}=\left\{y^{*} \in\right.$ $\left.H_{1}: 0 \in M_{1}^{y} y^{*}, \tilde{y}=T y^{*} \in H_{2}: 0 \in M_{2}^{y} \widetilde{y}\right\}$. Assume that
$\mathscr{F}^{x}=\mathcal{S}^{x} \cap\left(\cap_{i=1}^{N} V I\left(C, B_{i}^{x}\right)\right) \neq \varnothing \quad$ and $\mathscr{F}^{y}=\mathcal{S}^{y} \cap\left(\cap_{i=1}^{N}\right.$ $\left.V I\left(C, B_{i}^{y}\right)\right) \neq \varnothing$. Let $\left\{x_{n}\right\}$ and $\left\{y_{n}\right\}$ be sequences generated by $x_{1}, y_{1} \in H_{1}$ and

$$
\left\{\begin{array}{l}
x_{n+1}=\delta_{n} x_{n}+\sigma_{n} P_{C}\left(I-\mu_{n}^{x} \sum_{i=1}^{N} a_{i}^{x} B_{i}^{x}\right) x_{n}+\eta_{n}\left(\alpha_{n} f\left(y_{n}\right)+\left(1-\alpha_{n}\right) J_{\lambda_{1}^{x}}^{M_{1}^{x}}\left(x-\gamma^{x} T^{*}\left(I-J_{\lambda_{2}^{x}}^{M_{x}^{x}}\right) T x_{n}\right),\right. \\
y_{n+1}=\delta_{n} y_{n}+\sigma_{n} P_{C}\left(I-\mu_{n}^{x} \sum_{i=1}^{N} a_{i}^{y} B_{i}^{y}\right) y_{n}+\eta_{n}\left(\alpha_{n} g\left(x_{n}\right)+\left(1-\alpha_{n}\right) J_{\lambda_{1}^{y}}^{M_{1}^{y}}\left(y-\gamma^{y} T^{*}\left(I-J_{\lambda_{2}^{y}}^{M_{2}^{y}}\right) T y_{n}\right),\right.
\end{array}\right.
$$

for all $n \geq 1$, where $\left\{\delta_{n}\right\},\left\{\sigma_{n}\right\},\left\{\eta_{n}\right\},\left\{\alpha_{n}\right\} \subseteq[0,1]$ with $\delta_{n}+\sigma_{n}+\eta_{n}=1, \quad\left\{a_{1}^{x}, a_{2}^{x}, \ldots a_{N}^{x}\right\},\left\{a_{1}^{y}, a_{2}^{y}, \ldots, a_{N}^{y}\right\} \subset(0,1)$, $\lambda_{i}^{x}, \lambda_{i}^{y} \in(0, \infty)$ for all $i=1,2$, and $0<\gamma^{x}, \gamma^{y}<1 / L$ with $L$ being a spectral radius of $T^{*} T$. Assume the following conditions hold:

(1) $\sum_{n=1}^{\infty} \mu_{n}^{x}<\infty, \sum_{n=1}^{\infty} \mu_{n}^{y}<\infty$, and $0<a<\mu_{n}^{x} \leq 2 \overline{\beta_{x}}$, $0<b<\mu_{n}^{y} \leq 2 \overline{\beta_{y}}$, for some $a, b \in \mathbb{R}$.

(2) $\sum_{n=1}^{\infty} \alpha_{n}=\infty, \lim _{n \longrightarrow \infty} \alpha_{n}=0$.

(3) $\sum_{n=1}^{N} a_{i}^{x}=\sum_{n=1}^{N} a_{i}^{y}=1$.

(4) $0<\bar{a} \leq \delta_{n}, \sigma_{n}, \eta_{n} \leq \bar{b}<1$, for all $n \in \mathbb{N}$, for some $\bar{a}, \bar{b}>0$.

(5) $\sum_{n=1}^{\infty}\left|\delta_{n+1}-\delta_{n}\right|, \sum_{n=1}^{\infty}\left|\sigma_{n+1}-\sigma_{n}\right|, \quad$ and $\sum_{n=1}^{\infty} \mid \alpha_{n+1}-$ $\alpha_{n} \mid<\infty$. Then, $\left\{x_{n}\right\}$ converges strongly to $\tilde{x}=P_{\mathscr{F}^{x}} f(\widetilde{y})$ and $\left\{y_{n}\right\}$ converges strongly to $\tilde{y}=P_{\mathscr{F}^{y}} g(\tilde{x})$.

\section{Applications}

In this section, by applying our main result in Theorem 2, we can prove strong convergence theorems for approximating the solution of the split convex minimization problems and split feasibility problems.

4.1. Split Convex Minimization Problems. Let $\varphi: H \longrightarrow \mathbb{R}$ be a convex and differentiable function and $\psi: H \longrightarrow(-\infty, \infty]$ be a proper convex and lower semicontinuous function. It is well known that if $\nabla \varphi$ is $1 / \alpha$-Lipschitz continuous, then it is $\alpha$-inverse strongly monotone, where $\nabla \varphi$ is the gradient of $\varphi$ (see [10]). It is also known that the subdifferential $\partial \psi$ of $\psi$ is maximal monotone (see [35]). Moreover,

$\varphi\left(x^{*}\right)+\psi\left(x^{*}\right)=\min _{x \in H}[\varphi(x)+\psi(x)] \Leftrightarrow 0 \in \nabla \varphi\left(x^{*}\right)+\partial \varphi\left(x^{*}\right)$.
Next, we consider the following the split convex minimization problem (SCMP): find

$x^{*} \in H_{1}, \quad$ such that $\varphi_{1}\left(x^{*}\right)+\psi_{1}\left(x^{*}\right)=\min _{x \in H_{1}}\left[\varphi_{1}(x)+\psi_{1}(x)\right]$

and such that $y^{*}=T x^{*} \in H_{2}$ solves

$$
\varphi_{2}\left(y^{*}\right)+\psi_{2}\left(y^{*}\right)=\min _{y \in H_{2}}\left[\varphi_{2}(y)+\psi_{2}(y)\right],
$$

where $T: H_{1} \longrightarrow H_{2}$ is a bounded linear operator with adjoint $T^{*}, \varphi_{i}$, and $\psi_{i}$ defined as above, for $i=1,2$. We denoted the set of all solutions of (64) and (65) by $\Theta$. That is, $\Theta=\left\{x^{*}\right.$ which solves (64): $T x^{*}$ solves $\left.(65)\right\}$.

If we set $A_{i}^{x}=\nabla \varphi_{i}^{x}, A_{i}^{y}=\nabla \varphi_{i}^{y}$, and $M_{i}^{x}=\partial \psi_{i}^{x}, M_{i}^{y}=$ $\partial \psi_{i}^{y}$, for $i=1,2$, in Theorem 2, then we get the strong convergence theorem for finding the common solution of the split convex minimization problems and the finite families of the variational inequality problems as follows.

Theorem 3. Let $H_{1}$ and $H_{2}$ be Hilbert spaces, and let $C$ be a nonempty closed convex subset of $H_{1}$. Let $T: H_{1} \longrightarrow H_{2}$ be a bounded linear operator, and let $f, g: H_{1} \longrightarrow H_{1}$ be $\rho_{f}, \rho_{g}$-contraction mappings with $\rho=\max \left\{\rho_{f}, \rho_{g}\right\}$. For $i=1,2$, let $\psi_{i}^{x}, \psi_{i}^{y}: H_{i} \longrightarrow(-\infty, \infty]$ be proper convex and lower semicontinuous functions, and let $\varphi_{i}^{x}, \varphi_{i}^{y}: H_{i} \longrightarrow \mathbb{R}$ be convex and differentiable function such that $\nabla \varphi_{i}^{x}$ and $\nabla \varphi_{i}^{y}$ be $1 / \alpha_{i}^{x}$-Lipschitz continuous and $1 / \alpha_{i}^{y}$-Lipschitz continuous, respectively. For $i=1,2, \ldots, N$, let $B_{i}^{x}, B_{i}^{y}: H_{1} \longrightarrow H_{1}$ be $\beta_{i}^{x}, \beta_{i}^{y}$-inverse strongly monotone with $\bar{\beta}_{x}=\min _{i=1,2, \ldots, N}\left\{\beta_{i}^{x}\right\}$ and $\bar{\beta}_{y}=\min _{i=1,2, \ldots, N}\left\{\beta_{i}^{y}\right\}$. Let $\widetilde{G}^{x}, \widetilde{G}^{y}: H_{1} \longrightarrow H_{1}$ be defined by $\widetilde{G}^{x} x=J_{\lambda_{1}^{x}}^{\partial \psi_{1}^{x}}\left(I-\lambda_{1}^{x} \nabla \varphi_{1}^{x}\right)\left(x-\gamma^{x} T^{*}\left(I-J_{\lambda_{2}^{x}}^{\partial} \quad \psi_{2}^{x}\left(I-\lambda_{2}^{x}\right.\right.\right.$ $\left.\left.\left.\nabla \varphi_{2}^{x}\right)\right) T x\right), \forall x \in H_{1}$, and $\widetilde{G}^{y} y=J_{\lambda_{1}^{y}}^{\partial \psi_{1}^{y}}\left(I-\lambda_{1}^{y} \nabla \quad \varphi_{1}^{y}\right)(y-$ $\left.\gamma^{y} T^{*}\left(I-J_{\lambda_{2}^{y}}^{\partial \psi_{2}^{y}}\left(I-\lambda_{2}^{y} \nabla \varphi_{2}^{y}\right)\right) T y\right), \forall y \in H_{1}$, respectively, where $\lambda_{i}^{x} \in\left(0,2 \alpha_{i}^{x}\right), \lambda_{i}^{y} \in\left(0,2 \alpha_{i}^{y}\right)$ and $0<\gamma^{x}, \gamma^{y}<1 / L$ with $L$ is a spectral radius of $T^{*} T$. Assume that $\mathscr{F}^{x}=\Theta^{x} \cap\left(\cap_{i=1}^{N}\right.$ 
$\left.V I\left(C, B_{i}^{x}\right)\right) \neq \varnothing$ and $\mathscr{F}^{y}=\Theta^{y} \cap\left(\cap_{i=1}^{N} V I\left(C, B_{i}^{y}\right)\right) \neq \varnothing$. Let $\left\{x_{n}\right\}$ and $\left\{y_{n}\right\}$ be sequences generated by $x_{1}, y_{1} \in H_{1}$ and

$$
\left\{\begin{array}{l}
x_{n+1}=\delta_{n} x_{n}+\sigma_{n} P_{C}\left(I-\mu_{n}^{x} \sum_{i=1}^{N} a_{i}^{x} B_{i}^{x}\right) x_{n}+\eta_{n}\left(\alpha_{n} f\left(y_{n}\right)+\left(1-\alpha_{n}\right) \widetilde{G}^{x} x_{n}\right), \\
y_{n+1}=\delta_{n} y_{n}+\sigma_{n} P_{C}\left(I-\mu_{n}^{y} \sum_{i=1}^{N} a_{i}^{y} B_{i}^{y}\right) y_{n}+\eta_{n}\left(\alpha_{n} g\left(x_{n}\right)+\left(1-\alpha_{n}\right) \widetilde{G}^{y} y_{n}\right),
\end{array}\right.
$$

for all $n \geq 1$ where $\left\{\delta_{n}\right\},\left\{\sigma_{n}\right\},\left\{\eta_{n}\right\},\left\{\alpha_{n}\right\} \subseteq[0,1]$ with $\delta_{n}+\sigma_{n}+\eta_{n}=1, \quad\left\{a_{1}^{x}, a_{2}^{x}, \ldots a_{N}^{x}\right\},\left\{a_{1}^{y}, a_{2}^{y}, \ldots, a_{N}^{y}\right\} \subset(0,1)$, and $\left\{\mu_{n}^{x}\right\},\left\{\mu_{n}^{y}\right\} \subset(0, \infty)$. Assume the following condition holds:

(1) $\sum_{n=1}^{\infty} \mu_{n}^{x}<\infty, \sum_{n=1}^{\infty} \mu_{n}^{y}<\infty, \quad$ and $0<a<\mu_{n}^{x} \leq 2 \overline{\beta_{x}}$, $0<b<\mu_{n}^{y} \leq 2 \overline{\beta_{y}}$, for some $a, b \in \mathbb{R}$.

(2) $\sum_{n=1}^{\infty} \alpha_{n}=\infty, \lim _{n \longrightarrow \infty} \alpha_{n}=0$.

(3) $\sum_{n=1}^{N} a_{i}^{x}=\sum_{n=1}^{N} a_{i}^{y}=1$.

(4) $0<\bar{a} \leq \delta_{n}, \sigma_{n}, \eta_{n} \leq \bar{b}<1$, for all $n \in \mathbb{N}$, for some $\bar{a}, \bar{b}>0$

(5) $\sum_{n=1}^{\infty}\left|\delta_{n+1}-\delta_{n}\right|, \quad \sum_{n=1}^{\infty}\left|\sigma_{n+1}-\sigma_{n}\right|$, and $\sum_{n=1}^{\infty} \mid \alpha_{n+1}-$ $\alpha_{n} \mid<\infty$. Then, $\left\{x_{n}\right\}$ converges strongly to $\tilde{x}=P_{\mathscr{F}^{x}} f(\tilde{y})$ and $\left\{y_{n}\right\}$ converges strongly to $\tilde{y}=P_{\mathscr{F}^{y}} g(\tilde{x})$.

4.2. The Split Feasibility Problem. Let $H_{1}$ and $H_{2}$ be two real Hilbert spaces. Let $C$ and $Q$ be the nonempty closed convex subset of $H_{1}$ and $H_{2}$, respectively. The split feasibility problem (SFP) is to find

$$
\text { a point } x \in C, \quad \text { such that } A x \in Q \text {. }
$$

The set of all solutions (SFP) is denoted by $\Psi=\{x \in C: A x \in Q\}$. This problem was introduced by Censor and Elfving [8] in 1994. The split feasibility problem was investigated extensively as a widely important tool in many fields such as signal processing, intensity-modulated radiation therapy problems, and computer tomography (see [36-38] and the references therein).

Let $H$ be a real Hilbert space, and let $h$ be a proper lower semicontinuous convex function of $H$ into $(-\infty,+\infty]$. The subdifferential $\partial h$ of $h$ is defined by $\partial h(x)=\{z \in H: h(x)+\langle z, u-x\rangle \leq h(u), \forall u \in H\}$ for all $x \in H$. Then, $\partial h$ is a maximal monotone operator [39]. Let $C$ be a nonempty closed convex subset of $H$, and let $i_{C}$ be the indicator function of $C$, i.e., $i_{C}(x)=0$ if $x \in C$ and $i_{C}(x)=$ $\infty$ if $x \notin C$. Then, $i_{C}$ is a proper, lower semicontinuous and convex function on $H$, and so the subdifferential $\partial i_{C}$ of $i_{C}$ is a maximal monotone operator. Then, we can define the resolvent operator $J_{\lambda}^{\partial i_{C}}$ of $\partial i_{C}$ for $\lambda>0$, by $J_{\lambda}^{\partial i_{C}}(x)=\left(I+\lambda \partial i_{C}\right)^{-1}(x)$, for all $x \in H$.

Recall that the normal cone $N_{C}(u)$ of $C$ at a point $u$ in $H$ is defined by $N_{C}(u)=\{z \in H:\langle z, u-v \leq 0\rangle, \forall v \in C\}$ if $u \in C$ and $N_{C}(u)=\varnothing$ if $u \notin C$. We note that $\partial i_{C}=N_{C}$, and for $\lambda>0$, we have that $u=J_{\lambda}^{\partial i_{C}} x$ if and only if $u=P_{C} x$ (see [31]).

Setting $M_{1}=\partial i_{C}, M_{2}=\partial i_{Q}$, and in (SMVI) (4) and (5), then (SMVI) (4) and (5) are reduced to the split feasibility problem (SFP) (67)

Now, by applying Theorem 2, we get the following strong convergence theorem to approximate a common solution of SFP (67) and a finite family of variational inequality problems.

Theorem 4. Let $H_{1}$ and $H_{2}$ be Hilbert spaces, and let $C$ and $Q$ be the nonempty closed convex subset of $\mathrm{H}_{1}$ and $\mathrm{H}_{2}$, respectively. Let $T: H_{1} \longrightarrow H_{2}$ be a bounded linear operator with adjoint $T^{*}$, and let $f, g: H_{1} \longrightarrow H_{1}$ be $\rho_{f}, \rho_{g}$-contraction mappings with $\rho=\max \left\{\rho_{f}, \rho_{g}\right\}$. For $i=1,2, \ldots, N$, let $B_{i}^{x}, B_{i}^{y}: H_{1} \longrightarrow H_{1}$ be $\beta_{i}^{x}, \beta_{i}^{y}$-inverse strongly monotone with $\bar{\beta}_{x}=\min _{i=1,2, \ldots, N}\left\{\beta_{i}^{x}\right\}$ and $\bar{\beta}_{y}=\min _{i=1,2, \ldots, N}\left\{\beta_{i}^{y}\right\}$. Assume that $\mathscr{F}^{x}=\Psi^{x} \cap\left(\cap \cap_{i=1}^{N} V I\left(C, B_{i}^{x}\right)\right) \neq \varnothing$ and $\mathscr{F}^{y}=\Psi^{y} \cap\left(\cap_{i=1}^{N} V I\left(C, B_{i}^{y}\right)\right) \neq \varnothing$. Let $\left\{x_{n}\right\}$ and $\left\{y_{n}\right\}$ be sequences generated by $x_{1}, y_{1} \in H_{1}$ and

$$
\left\{\begin{array}{l}
x_{n+1}=\delta_{n} x_{n}+\sigma_{n} P_{C}\left(I-\mu_{n}^{x} \sum_{i=1}^{N} a_{i}^{x} B_{i}^{x}\right) x_{n}+\eta_{n}\left(\alpha_{n} f\left(y_{n}\right)+\left(1-\alpha_{n}\right) P_{C}\left(x-\gamma^{x} T^{*}\left(I-P_{Q}\right) T x_{n}\right)\right), \\
y_{n+1}=\delta_{n} y_{n}+\sigma_{n} P_{C}\left(I-\mu_{n}^{x} \sum_{i=1}^{N} a_{i}^{y} B_{i}^{y}\right) y_{n}+\eta_{n}\left(\alpha_{n} g\left(x_{n}\right)+\left(1-\alpha_{n}\right) P_{C}\left(y-\gamma^{y} T^{*}\left(I-P_{Q}\right) T y_{n}\right),\right.
\end{array}\right.
$$


for all $n \geq 1$, where $\left\{\delta_{n}\right\},\left\{\sigma_{n}\right\},\left\{\eta_{n}\right\},\left\{\alpha_{n}\right\} \subseteq[0,1]$ with $\delta_{n}+\sigma_{n}+\eta_{n}=1, \quad\left\{a_{1}^{x}, a_{2}^{x}, \ldots, a_{N}^{x}\right\},\left\{a_{1}^{y}, a_{2}^{y}, \ldots, a_{N}^{y}\right\} \subset(0,1)$, $\left\{\mu_{n}^{x}\right\},\left\{\mu_{n}^{y}\right\} \subset(0, \infty), \lambda_{i}^{x}, \lambda_{i}^{y} \in(0, \infty)$ for all $i=1,2$, and $0<\gamma^{x}, \gamma^{y}<1 / L$ with $L$ being a spectral radius of $T^{*} T$. Assume the following condition holds:

(1) $\sum_{n=1}^{\infty} \mu_{n}^{x}<\underline{\infty}, \sum_{n=1}^{\infty} \mu_{n}^{y}<\infty$, and $0<a<\mu_{n}^{x} \leq 2 \overline{\beta_{x}}, 0<$ $b<\mu_{n}^{y} \leq 2 \overline{\beta_{y}}$, for some $a, b \in \mathbb{R}$.

(2) $\sum_{n=1}^{\infty} \alpha_{n}=\infty, \lim _{n \longrightarrow \infty} \alpha_{n}=0$.

(3) $\sum_{n=1}^{N} a_{i}^{x}=\sum_{n=1}^{N} a_{i}^{y}=1$.

(4) $0<\bar{a} \leq \delta_{n}, \sigma_{n}, \eta_{n} \leq \bar{b}<1$, for all $n \in \mathbb{N}$, for some $\bar{a}, \bar{b}>0$.

(5) $\sum_{n=1}^{\infty}\left|\delta_{n+1}-\delta_{n}\right|, \quad \sum_{n=1}^{\infty}\left|\sigma_{n+1}-\sigma_{n}\right|$, and $\sum_{n=1}^{\infty} \mid \alpha_{n+1}$ $-\alpha_{n} \mid<\infty$. Then, $\left\{x_{n}\right\}$ converges strongly to $\tilde{x}=P_{\mathscr{F}^{x}} f(\tilde{y})$ and $\left\{y_{n}\right\}$ converges strongly to $\tilde{y}=P_{\mathscr{F}^{y}} g(\tilde{x})$.

Proof. Set $M_{1}^{x}=\partial i_{C}, M_{1}^{y}=\partial i_{C}, M_{2}^{x}=\partial i_{Q}, M_{2}^{y}=\partial i_{Q}$, and $A_{i}^{x}=0$ and $A_{i}^{y}=0$ in Theorem 2. Then, we get the result.

The split feasibility problem is a significant part of the split monotone variational inclusion problem. It is extensively used to solve practical problems in numerous situations. Many excellent results have been obtained. In what follows, an example of a signal recovery problem is introduced.

Example 2. In signal recovery, compressed sensing can be modeled as the following under-determined linear equation system:

$$
y=A x+\delta
$$

where $x \in \mathbb{R}^{N}$ is a vector with $m$ non-zero components to be recovered, $y \in \mathbb{R}^{M}$ is the observed or measured data with noisy $\delta$, and $A: \mathbb{R}^{N} \longrightarrow \mathbb{R}^{M}(M<N)$ is a bounded linear observation operator. An essential point of this problem is that the signal $x$ is sparse; that is, the number of nonzero elements in the signal $x$ is much smaller than the dimension of the signal $x$. To solve this situation, a classical model, convex constraint minimization problem, is used to describe the above problem. It is known that problem (69) can be seen as solving the following LASSO problem [40]:

$$
\min _{x \in \mathbb{R}^{N}} \frac{1}{2}\|y-A x\|_{2}^{2}, \quad \text { subject to }\|x\|_{1} \leq t
$$

where $t>0$ is a given constant and $\|\cdot\|_{1}$ is $\ell_{1}$ norm. In particular, LASSO problem (70) is equivalent to the split feasibility problem (SFP) (67) when $C=\left\{x \in \mathbb{R}^{N}:\|x\|_{1} \leq t\right\}$ and $Q=\{y\}$.

\section{Numerical Examples}

In this section, we give some examples for supporting Theorem 2. In example 3, we give the computer programming to support our main result.

Example 3. Let $\mathbb{R}$ be a set of real number and $H_{1}=H_{2}=\mathbb{R}^{2}$. Let $C=[-20,20] \times[-20,20]$, and let $\langle\cdot, \cdot\rangle: \mathbb{R}^{2} \times \mathbb{R}^{2} \longrightarrow \mathbb{R}$ be inner product defined by $\langle x, y\rangle=x \cdot y=x_{1} y_{1}+x_{2} y_{2}$, for all $x=\left(x_{1}, x_{2}\right) \in \mathbb{R}^{2}$ and $y=\left(y_{1}, y_{2}\right) \in \mathbb{R}^{2}$ and the usual norm $\|\cdot\|: \mathbb{R}^{2} \longrightarrow \mathbb{R}$ given by $\|x\|=\sqrt{x_{1}^{2}+x_{2}^{2}}$, for all $x=\left(x_{1}, x_{2}\right) \in \mathbb{R}^{2}$. Let $T: \mathbb{R}^{2} \longrightarrow \mathbb{R}^{2}$ be defined by $T x=\left(2 x_{1}, 2 x_{2}\right) \quad$ for all $\quad x=\left(x_{1}, x_{2}\right) \in \mathbb{R}^{2} \quad$ and $T^{*}: \mathbb{R}^{2} \longrightarrow \mathbb{R}^{2}$ be defined by $T^{*} z=\left(2 z_{1}, 2 z_{2}\right)$ for all $z=\left(z_{1}, z_{2}\right) \in \mathbb{R}^{2}$. Let $M_{1}^{x}, M_{1}^{y}, M_{2}^{x}, M_{2}^{y}: \mathbb{R}^{2} \longrightarrow 2^{\mathbb{R}^{2}}$ be defined by $M_{1}^{x} x=\left\{\left(3 x_{1}-2,3 x_{2}-2\right)\right\}, M_{1}^{y} x=\left\{\left(2 x_{1}\right.\right.$, $\left.\left.2 x_{2}\right)\right\}, M_{2}^{x}=\left\{\left(\left(x_{1} / 3\right)+2, \quad\left(x_{2} / 3\right)+2\right)\right\}$, and $M_{2}^{y} x=\left\{\left(\left(x_{1} /\right.\right.\right.$ $\left.\left.3)+3,\left(x_{2} / 3\right)+3\right)\right\}$, respectively, for all $x=\left(x_{1}, x_{2}\right) \in \mathbb{R}^{2}$. Let the mapping $A_{1}^{x}, A_{1}^{y}, A_{2}^{x}, A_{2}^{y}: \mathbb{R}^{2} \longrightarrow \mathbb{R}^{2}$ be defined by $A_{1}^{x} x=\left(\left(x_{1}-3\right) / 2,\left(x_{2}-3\right) / 2\right), A_{1}^{y} x=\left(x_{1}+6, x_{2}+6\right), A_{2}^{x} x$ $=\left(\left(x_{1}-2\right) / 3,\left(x_{2}-2\right) / 3\right)$, and $A_{2}^{y}=\left(\left(x_{1}-1\right) / 3,\left(x_{2}-1\right) / 3\right)$, respectively, for all $x=\left(x_{1}, x_{2}\right) \in \mathbb{R}^{2}$. For every $i=1,2, \ldots, N$, let the mappings $B_{i}^{x}, B_{i}^{y}: \mathbb{R}^{2} \longrightarrow \mathbb{R}^{2}$ be defined by $B_{i}^{x} x=\left(\left(x_{1}-1\right) / 3 i,\left(x_{2}-1\right) / 3 i\right)$ and $B_{i}^{y} x=$ $\left(\left(x_{1}+2\right) / 5 i,\left(x_{2}+2\right) / 5 i\right), \quad$ respectively, for all $x=\left(x_{1}, x_{2}\right) \in \mathbb{R}^{2}$, and let $a_{i}^{x}=\left(2 / 3^{i}+1 / N 3^{N}\right)$ and $a_{i}^{y}=\left(4 / 5^{i}+1 / N 5^{N}\right)$. Let the mappings $f, g: \mathbb{R}^{2} \longrightarrow \mathbb{R}^{2}$ be defined by $f(x)=\left(x_{1} / 7, x_{2} / 7\right)$ and $g(x)=\left(x_{1} / 9, x_{2} / 9\right)$, respectively, for all $x=\left(x_{1}, x_{2}\right) \in \mathbb{R}^{2}$.

Choose $\quad \gamma^{x}$ and $\gamma^{y}=0.1, \quad \lambda_{1}^{x}=2, \lambda_{1}^{y}=1.2, \lambda_{2}^{x}=0.1$, and $\lambda_{2}^{y}=1.9$. Setting $\left\{\delta_{n}\right\}=\{n /(9 n+3)\}, \quad\left\{\sigma_{n}\right\}=\{(4 n+$ $(2 / 3)) /(9 n+3)\}, \quad\left\{\eta_{n}\right\}=\{(4 n+(7 / 3)) /(9 n+3)\}, \quad\left\{\alpha_{n}\right\}=$ $\{1 / 20 n\}, \quad\left\{\mu_{n}^{x}\right\}=\left\{1 / 7 n^{2}\right\}, \quad$ and $\quad\left\{\mu_{n}^{y}\right\}=\left\{1 / 5 n^{2}\right\}$. Let $x_{1}=\left(x_{1}^{1}, x_{1}^{2}\right)$ and $y_{1}=\left(y_{1}^{1}, y_{1}^{2}\right) \in \mathbb{R}^{2}$, and let the sequences $\left\{x_{n}\right\}$ and $\left\{y_{n}\right\}$ be generated by (21) as follows: 
TABLe 1: Values of $\left\{x_{n}\right\}$ and $\left\{y_{n}\right\}$ with initial values $x_{1}=(-10,10), y_{1}=(-10,10)$, and $n=N=50$.

\begin{tabular}{ccc}
\hline$n$ & $x_{n}=\left(x_{n}^{1}, x_{n}^{2}\right)$ & $y_{n}=\left(y_{n}^{1}, y_{n}^{2}\right)$ \\
\hline 1 & $(-10.000000,10.000000)$ & $(-10.000000,10.000000)$ \\
2 & $(-4.093342,5.126154)$ & $(-5.481385,3.338182)$ \\
3 & $(-1.544910,3.037093)$ & $(-3.639877,0.575101)$ \\
4 & $(-0.307347,2.029037)$ & $(-2.789106,-0.719370)$ \\
$\vdots$ & $\vdots$ & $\vdots$ \\
30 & $(0.998745,0.996821)$ & $(-1.996611,-1.998243)$ \\
$\vdots$ & $\vdots$ & $\vdots$ \\
47 & $(0.998973,0.998235)$ & $(-1.998394,-1.999022)$ \\
48 & $(0.998986,0.998280)$ & $(-1.998454,-1.999055)$ \\
49 & $(0.999000,0.998324)$ & $(-1.998511,-1.999087)$ \\
50 & $(0.999013,0.998365)$ & $(-1.998566,-1.999118)$ \\
\hline
\end{tabular}

$$
\left\{\begin{array}{l}
x_{n+1}=\frac{n}{9 n+3} x_{n}+\frac{4 n+(2 / 3)}{9 n+3} P_{C}\left(I-\frac{1}{7 n^{2}} \sum_{i=1}^{N}\left(\frac{2}{3^{i}}+\frac{1}{N 3^{N}}\right) B_{i}^{x}\right) x_{n}+\frac{4 n+7 / 3}{9 n+3}\left(\frac{1}{20 n} f\left(y_{n}\right)+\frac{20 n-1}{20 n} \widetilde{G}^{x} x_{n}\right) \\
y_{n+1}=\frac{n}{9 n+3} y_{n}+\frac{4 n+(2 / 3)}{9 n+3} P_{C}\left(I-\frac{1}{5 n^{2}} \sum_{i=1}^{N}\left(\frac{4}{5^{i}}+\frac{1}{N 5^{N}}\right) B_{i}^{y}\right) y_{n}+\frac{4 n+7 / 3}{9 n+3}\left(\frac{1}{20 n} g\left(x_{n}\right)+\frac{20 n-1}{20 n} \widetilde{G}^{y} y_{n}\right) \\
\widetilde{G}^{x} x_{n}=J_{2}^{M_{1}^{x}}\left(I-2 A_{1}^{x}\right)\left(x-0.1 T^{*}\left(I-J_{0.1}^{M_{2}^{x}}\left(I-0.1 A_{2}^{x}\right)\right) T x_{n}\right) \\
\widetilde{G}^{y} y_{n}=J_{1.2}^{M_{1}^{y}}\left(I-1.2 A_{1}^{y}\right)\left(y-0.1 T^{*}\left(I-J_{1.9}^{M_{2}^{y}}\left(I-1.9 A_{2}^{y}\right)\right) T y_{n}\right)
\end{array}\right.
$$

for all $n \geq 1$, where $x_{n}=\left(x_{n}^{1}, x_{n}^{2}\right)$ and $y_{n}=\left(y_{n}^{1}, y_{n}^{2}\right)$. By the definition of $M_{i}^{x}, M_{i}^{y}, A_{i}^{x}$, and $A_{i}^{y}$, for all $i=1,2, B_{i}^{x}$ and $B_{i}^{y}$, for all $i=1,2, \ldots, N$, and $f$ and $g$, we have that $(1,1) \in \Omega^{x} \cap\left(\cap_{i=1}^{N} V I\left(C, B_{i}^{x}\right)\right) \quad$ and $\quad(-2,-2) \in \Omega^{y} \cap$ $\left(\cap_{i=1}^{N} V I\left(C, B_{i}^{y}\right)\right)$. Also, it is easy to see that all parameters satisfy all conditions in Theorem 2 . Then, by Theorem 2 , we can conclude that the sequence $\left\{x_{n}\right\}$ converges strongly to $(1,1)$ and $\left\{y_{n}\right\}$ converges strongly to $(-2,-2)$.

Table 1 and Figure 1 show the numerical results of $\left\{x_{n}\right\}$ and $\left\{y_{n}\right\}$ where $x_{1}=(-10,10), \quad y_{1}=(-10,10)$, and $n=N=50$.

Next, in Example 4, we only show an example in infinitedimensional Hilbert space for supporting Theorem 2. We omit the computer programming.

Example 4. Let $H_{1}=H_{2}=C=\ell_{2}$ be the linear space whose elements consist of all 2-summable sequence $\left(x_{1}, x_{2}, \ldots, x_{j}, \ldots\right)$ of scalars, i.e.,

$\ell_{2}=\left\{x: x=\left(x_{1}, x_{2}, \ldots, x_{j}, \ldots\right)\right.$ and $\left.\sum_{j=1}^{\infty}\left|x_{j}\right|^{2}<\infty\right\}$, with an inner product $\langle\cdot, \cdot\rangle: \ell_{2} \times \ell_{2} \longrightarrow \mathbb{R}$ defined by $\langle x, y\rangle=\sum_{j=1}^{\infty} x_{j} y_{j}$, where $x=\left\{x_{j}\right\}_{j=1}^{\infty}$ and $y=\left\{y_{j}\right\}_{j=1}^{\infty} \in \ell_{2}$, and a norm $\|\cdot\|: \ell_{2} \longrightarrow \mathbb{R}$ defined by $\|x\|_{2}=\left(\sum_{j=1}^{\infty}\left|x_{j}\right|^{2}\right)^{1 / 2}$ where $x=\left\{x_{j}\right\}_{j=1}^{\infty} \in \ell_{2}$. Let $T: \ell_{2} \longrightarrow \ell_{2}$ be defined by $T x=$ $\left(x_{1} / 2, x_{2} / 2, \ldots, x_{j} / 2, \ldots\right)$ for all $x=\left\{x_{j}\right\}_{j=1}^{\infty} \in \ell_{2}$. And, $T^{*}: \ell_{2} \longrightarrow \ell_{2}$ be defined by $T x=\left(z_{1} / 2, z_{2} / 2, \ldots, z_{j} / 2, \ldots\right)$ for all $x=\left\{z_{j}\right\}_{j=1}^{\infty} \in \ell_{2}$. Let $M_{1}^{x}, M_{1}^{y}, M_{2}^{x}, M_{2}^{y}: \ell_{2} \longrightarrow \ell_{2}$ be defined by $M_{1}^{x} x=\left\{\left(2 x_{1}, 2 x_{2}, \ldots, 2 x_{j}, \ldots\right)\right\}, M_{1}^{y} x=\left\{\left(x_{1}-\right.\right.$ $\left.\left.1, x_{2}-1, \ldots, x_{j}-1, \ldots\right)\right\}, M_{2}^{x}=\left\{3 x_{1}, 3 x_{2}, \ldots, 3 x_{j}, \ldots\right\}$, and $M_{2}^{y} x=\left\{\left(2 x_{1}-1,2 x_{2}-1, \ldots, 2 x_{j}-1, \ldots\right)\right\}, \quad$ respectively, for all $x=\left\{x_{j}\right\}_{j=1}^{\infty} \in \ell_{2}$. Let the mapping $A_{1}^{x}, A_{1}^{y}, A_{2}^{x}, A_{2}^{y}$ : $\ell_{2} \longrightarrow \ell_{2}$ be defined by $A_{1}^{x} x=\left(x_{1} / 3, x_{2} / 3, \ldots, x_{j} / 3, \ldots\right)$, $A_{1}^{y} x=\left(\left(x_{1}-1\right) / 2,\left(x_{2}-1\right) / 2, \ldots,\left(x_{j}-1\right) / 2, \ldots\right), \quad A_{2}^{x} x$ $=\left(x_{1} / 4, x_{2} / 4, \ldots, x_{j} / 4, \ldots\right)$, and $A_{2}^{y}=\left(\left(2 x_{1}-1\right) / 3,\left(2 x_{2}-\right.\right.$ $\left.1) / 3, \ldots,\left(2 x_{j}-1\right) / 3\right)$ respectively, for all $x=\left\{x_{j}\right\}_{j=1}^{\infty} \in \ell_{2}$. For every $i=1,2, \ldots, N$, let the mappings $B_{i}^{x}, B_{i}^{y}: \ell_{2} \longrightarrow \ell_{2}$ be defined by $B_{i}^{x} x=\left(2 x_{1} / 3 i, 2 x_{2} / 3 i, \ldots, 2 x_{j} / 3 i, \ldots\right)$ and $B_{i}^{y} x$ $=\left(\left(2 x_{1}-1\right) / 4 i,\left(2 x_{2}-1\right) / 4 i, \ldots,\left(2 x_{j}-1\right) / 4 i\right)$, respectively, 


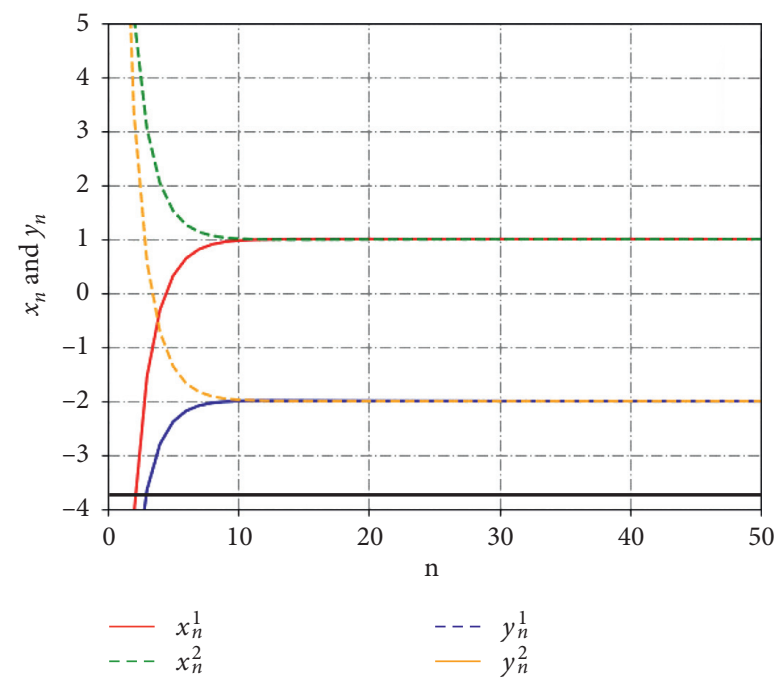

FIGURE 1: Convergence of $\left\{x_{n}\right\}$ and $\left\{y_{n}\right\}$ with initial values $x_{1}=(-10,10), y_{1}=(-10,10)$, and $n=N=50$.

for all $x=\left\{x_{j}\right\}_{j=1}^{\infty} \in \ell_{2}$, and let $a_{i}^{x}=\left(5 / 6^{i}+1 / N 6^{N}\right)$ and $a_{i}^{y}=\left(7 / 8^{i}+1 / N 8^{N}\right)$. Let the mappings $f, g: \ell_{2} \longrightarrow \ell_{2}$ be defined by $f(x)=\left(x_{1} / 5, x_{2} / 5, \ldots, x_{j} / 5, \ldots\right), g(x)=$ $\left(x_{1} / 4, x_{2} / 4, \ldots, x_{j} / 4, \ldots\right), \quad$ respectively, for all $x=\left\{x_{j}\right\}_{j=1}^{\infty} \in \ell_{2}$.
Let $\lambda_{1}^{x}=1, \lambda_{1}^{y}=1, \lambda_{2}^{x}=0.5$, and $\lambda_{2}^{y}=2$. Since $L=1 / 4$, we choose $\gamma^{x}$ and $\gamma^{y}=0.5$. Let $x_{1}=\left(x_{1}^{1}, x_{1}^{2}, \ldots, x_{1}^{j}, \ldots\right)$ and $y_{1}=\left(y_{1}^{1}, y_{1}^{2}, \ldots, y_{1}^{j}, \ldots\right) \in \ell_{2}$, and let $\left\{x_{n}\right\}$ and $\left\{y_{n}\right\}$ be the sequences generated by (2) as follows:

$$
\left\{\begin{array}{l}
x_{n+1}=\frac{n}{5 n+2} x_{n}+\frac{2 n+2 / 3}{5 n+2} P_{C}\left(I-\frac{1}{3 n^{2}} \sum_{i=1}^{N}\left(\frac{5}{6^{i}}+\frac{1}{N 6^{N}}\right) B_{i}^{x}\right) x_{n}+\frac{2 n+4 / 3}{5 n+3}\left(\frac{1}{10 n} f\left(y_{n}\right)+\frac{10 n-1}{20 n} \widetilde{G}^{x} x_{n}\right) \\
y_{n+1}=\frac{n}{5 n+2} y_{n}+\frac{2 n+2 / 3}{5 n+2} P_{C}\left(I-\frac{1}{4 n^{2}} \sum_{i=1}^{N}\left(\frac{7}{8^{i}}+\frac{1}{N 8^{N}}\right) B_{i}^{y}\right) y_{n}+\frac{2 n+4 / 3}{5 n+2}\left(\frac{1}{10 n} g\left(x_{n}\right)+\frac{10 n-1}{20 n} \widetilde{G}^{y} y_{n}\right) \\
\widetilde{G}^{x} x_{n}=J_{1}^{M_{1}^{x}}\left(I-A_{1}^{x}\right)\left(x-0.5 T^{*}\left(I-J_{0.5}^{M_{2}^{x}}\left(I-0.5 A_{2}^{x}\right)\right) T x_{n}\right) \\
\widetilde{G}^{y} y_{n}=J_{1}^{M_{1}^{y}}\left(I-A_{1}^{y}\right)\left(y-0.5 T^{*}\left(I-J_{2}^{M_{2}^{y}}\left(I-2 A_{2}^{y}\right)\right) T y_{n}\right)
\end{array}\right.
$$

for all $n \geq 1$, where $x_{n}=\left(x_{n}^{1}, x_{n}^{2}, \ldots, x_{n}^{j}, \ldots\right)$ and $y_{n}=$ $\left(y_{n}^{1}, y_{n}^{2}, \ldots, y_{n}^{j}, \ldots\right) . \quad$ It is easy to see that $M_{i}^{x}$, $M_{i}^{y}, A_{i}^{x}$, and $A_{i}^{y}, \forall i=1,2, B_{i}^{x}$ and $B_{i}^{y}, \forall i=1,2, \ldots, N, T, f, g$, and all parameters satisfy Theorem 2 . Furthermore, we have that $0 \in \Omega^{x} \cap\left(\cap_{i=1}^{N} V I\left(C, B_{i}^{x}\right)\right)$ and $1 \in \Omega^{y} \cap\left(\cap_{i=1}^{N} V I(C\right.$, $\left.\left.B_{i}^{y}\right)\right)$. Then, by Theorem 2 , we can conclude that the sequence $\left\{x_{n}\right\}$ converges strongly to 0 and $\left\{y_{n}\right\}$ converges strongly to 1 .

\section{Conclusion}

(1). Table 1 and Figure 1 in Example 3 show that the sequence $\left\{x_{n}\right\}$ converges to $(1,1) \in \Omega^{x} \cap$ $\left(\cap_{i=1}^{N} V I\left(C, B_{i}^{x}\right)\right)$ and $\left\{y_{n}\right\}$ converges to $(-2,-2) \in \Omega^{y} \cap\left(\cap_{i=1}^{N} V I\left(C, B_{i}^{y}\right)\right)$

(2) Example 4 is an example in infinite-dimensional Hilbert space for supporting Theorem 2 
(3) Theorem 2 guarantees the convergence of $\left\{x_{n}\right\}$ and $\left\{y_{n}\right\}$ in Example 3 and Example 4

\section{Data Availability}

No data were used to support this study.

\section{Conflicts of Interest}

The authors declare no conflicts of interest.

\section{Acknowledgments}

The authors would like to thank the Faculty of Science and Technology, Rajamangala University of Technology Thanyaburi (RMUTT), Thailand, for the financial support.

\section{References}

[1] A. Moudafi, "Split monotone variational inclusions," Journal of Optimization Theory and Applications, vol. 150, no. 2, pp. 275-283, 2011.

[2] Y. Censor, A. Gibali, and S. Reich, "Algorithms for the split variational inequality problem," Numerical Algorithms, vol. 59, pp. 301-323, 2012.

[3] A. S. Alofi, S. M. Alsulami, and W. Takahashi, "The split common null point problem and halpern-type strong convergence theorem in hilbert spaces," Journal of Nonlinear and Convex Analysis, vol. 16, no. 5, pp. 775-789, 2015.

[4] C. Byrne, Y. Censor, A. Gibali, and S. Reich, "The split common null point problem," Journal Nonlinear Convex Anal, vol. 13, no. 4, pp. 759-775, 2012.

[5] H. Che and M. Li, "The conjugate gradient method for split variational inclusion and constrained convex minimization problems," Applied Mathematics and Computation, vol. 290, pp. $426-438,2016$.

[6] L.-J. Lin, Y.-D. Chen, and C.-S. Chuang, "Solutions for a variational inclusion problem with applications to multiple sets split feasibility problems," Fixed Point Theory and Applications, vol. 2013, no. 1, pp. 1-21, 2013.

[7] S. Takahashi and W. Takahashi, "The split common null point problem and the shrinking projection method in banach spaces," Optimization, vol. 65, no. 2, pp. 281-287, 2016.

[8] Y. Censor and T. Elfving, "A multiprojection algorithm using bregman projections in a product space," Numerical Algorithms, vol. 8, no. 2, pp. 221-239, 1994.

[9] C. Byrne, "Iterative oblique projection onto convex sets and the split feasibility problem," Inverse Problems, vol. 18, no. 2, pp. 441-453, 2002.

[10] C. Byrne, "A unified treatment of some iterative algorithms in signal processing and image reconstruction," Inverse Problems, vol. 20, no. 1, pp. 103-120, 2003.

[11] Z.-T. Yu, L.-J. Lin, and C.-S. Chuang, "Mathematical programming with multiple sets split monotone variational inclusion constraints," Fixed Point Theory and Applications, vol. 2014, no. 1, pp. 1-27, 2014.

[12] Q. H. Ansari and A. Rehan, "An iterative method for split hierarchical monotone variational inclusions," Fixed Point Theory and Applications, vol. 2015, no. 1, pp. 1-10, 2015.

[13] K. Kazmi, S. Rizvi, and R. Ali, "A hybrid-extragradient iterative method for split monotone variational inclusion, mixed equilibrium problem and fixed point problem for a nonexpansive mapping," Journal of the Nigerian Mathematical Society, vol. 35, no. 2, pp. 312-338, 2016.

[14] Y. Shehu and F. U. Ogbuisi, "An iterative method for solving split monotone variational inclusion and fixed point problems," Revista de la Real Academia de Ciencias Exactas, Físicas y Naturales. Serie A, Matemáticas, vol. 110, no. 2, pp. 503-518, 2016.

[15] K. R. Kazmi, R. Ali, and M. Furkan, "Hybrid iterative method for split monotone variational inclusion problem and hierarchical fixed point problem for a finite family of nonexpansive mappings," Numerical Algorithms, vol. 79, no. 2, pp. 499-527, 2018.

[16] B. Chaloemyotphong and A. Kangtunyakarn, "Modified halpern iterative method for solving hierarchical problem and split combination of variational inclusion problem in hilbert space," Mathematics, vol. 7, no. 11, Article ID 1037, 2019.

[17] X. Zhao, J.-C. Yao, and Y. Yao, "A proximal algorithm for solving split monotone variational inclusions," University Politehnica of Bucharest Scientific, Bulletin-Series A-Applied Mathematics and Physics, vol. 82, no. 3, pp. 43-52, 2020.

[18] F. U. Ogbuisi and O. T. Mewomo, "Solving split monotone variational inclusion problem and fixed point problem for certain multivalued maps in hilbert spaces," Thai Journal of Mathematics, vol. 19, no. 2, pp. 503-520, 2021.

[19] Y. Yao, Y. Shehu, X.-H. Li, and Q.-L. Dong, "A method with inertial extrapolation step for split monotone inclusion problems,” Optimization, vol. 70, no. 4, pp. 741-761, 2021.

[20] Z. Yao, S. M. Kang, and H.-J. Li, "An intermixed algorithm for strict pseudo-contractions in hilbert spaces," Fixed Point Theory and Applications, vol. 2015, no. 1, Article ID 206, 2015.

[21] S. Suwannaut, "The s-intermixed iterative method for equilibrium problems," Thai Journal of Mathematics, pp. 60-74, 2018.

[22] S. Suwannaut, "The general intermixed iteration for equilibrium problems and variational inequality problems in hilbert spaces," Thai Journal of Mathematics, vol. 18, no. 3, pp. 1497-1518, 2020.

[23] W. Nazeer, M. Munir, and S. M. Kang, "An intermixed algorithm for three strict pseudo-contractions in hilbert spaces," Journal of Computational Analysis and Applications, vol. 24, pp. 1322-1333, 2018.

[24] L. O. Jolaoso, F. Ogbuisi, and O. Mewomo, "An intermixed algorithm for two strict pseudocontractions in q-uniformly smooth banach space," Nonlinear Studies, vol. 26, no. 1, pp. 27-41, 2019.

[25] A. Sripattanet and A. Kangtunyakarn, "Convergence theorem for solving a new concept of the split variational inequality problems and application," Revista de la Real Academia de Ciencias Exactas, Físicas y Naturales. Serie A, Matemáticas, vol. 114, no. 4, pp. 1-33, 2020.

[26] A. Sripattanet and A. Kangtunyakarn, "Convergence theorem of two sequences for solving the modified generalized system of variational inequalities and numerical analysis," Mathematics, vol. 7, no. 10, Article ID 916, 2019.

[27] K. Saechou and A. Kangtunyakarn, "An intermixed iteration for constrained convex minimization problem and split feasibility problem," Journal of Inequalities and Applications, vol. 2019, no. 1, Article ID 269, 2019.

[28] T. Suzuki, "Strong convergence theorems for infinite families of nonexpansive mappings in general banach spaces," Fixed Point Theory and Applications, vol. 2005, no. 1, pp. 1-21, 2005.

[29] K. Shimoji and W. Takahashi, "Strong convergence to common fixed points of infinite nonexpansive mappings and 
applications," Taiwanese Journal of Mathematics, vol. 5, no. 2, pp. 387-404, 2001.

[30] W. Takahashi, Nonlinear Functional Analysis, Yokohama Publishere, Yokohama, Japan, 2000.

[31] W. Takahashi, Introduction to Nonlinear and Convex Analysis, Yokohama Publishers, Yokohama, Japan, 2009.

[32] W. Khuangsatung and A. Kangtunyakarn, "The applications of modified generalized mixed equilibrium problems to nonlinear problems," Thai Journal of Mathematics, vol. 14, no. 3, pp. 771-796, 2016.

[33] S.-s. Zhang, J. H. W. Lee, and C. K. Chan, "Algorithms of common solutions to quasi variational inclusion and fixed point problems," Applied Mathematics and Mechanics, vol. 29, no. 5, pp. 571-581, 2008.

[34] H. K. Xu, "An iterative approach to quadratic optimization," Journal of Optimization Theory and Applications, vol. 116, no. 3, pp. 659-678, 2003.

[35] R. T. Rockafellar, "On the maximality of sums of nonlinear monotone operators," Transactions of the American Mathematical Society, vol. 149, no. 1, pp. 75-88, 1970.

[36] B. Qu and N. Xiu, "A note on the cq algorithm for the split feasibility problem," Inverse Problems, vol. 21, no. 5, pp. 1655-1665, 2005.

[37] Y. Censor, T. Bortfeld, B. Martin, and A. Trofimov, "A unified approach for inversion problems in intensity-modulated radiation therapy," Physics in Medicine and Biology, vol. 51, no. 10, pp. 2353-2365, 2006.

[38] Y. Censor, A. Motova, and A. Segal, "Perturbed projections and subgradient projections for the multiple-sets split feasibility problem," Journal of Mathematical Analysis and Applications, vol. 327, no. 2, pp. 1244-1256, 2007.

[39] R. Rockafellar, "On the maximal monotonicity of subdifferential mappings," Pacific Journal of Mathematics, vol. 33, no. 1, pp. 209-216, 1970.

[40] R. Tibshirani, "Regression shrinkage and selection via the lasso," Journal of the Royal Statistical Society: Series B, vol. 58, no. 1, pp. 267-288, 1996. 